TRANSACTIONS OF THE

AMERICAN MATHEMATICAL SOCIETY

Volume 349, Number 2, February 1997, Pages 763-788

S 0002-9947(97)01804-7

\title{
ENRICHED $P$-PARTITIONS
}

\author{
JOHN R. STEMBRIDGE
}

\begin{abstract}
An (ordinary) $P$-partition is an order-preserving map from a partially ordered set to a chain, with special rules specifying where equal values may occur. Examples include number-theoretic partitions (ordered and unordered, strict or unrestricted), plane partitions, and the semistandard tableaux associated with Schur's $S$-functions. In this paper, we introduce and develop a theory of enriched $P$-partitions; like ordinary $P$-partitions, these are order-preserving maps from posets to chains, but with different rules governing the occurrence of equal values. The principal examples of enriched $P$-partitions given here are the tableaux associated with Schur's $Q$-functions. In a sequel to this paper, further applications related to commutation monoids and reduced words in Coxeter groups will be presented.
\end{abstract}

\section{INTRODUCTION}

This is the first of a series of related papers on the combinatorics of reduced expressions in Coxeter groups, pattern avoidance, commutation monoids, and $P$ partitions. Our initial motivation for studying the title subject arose from investigations of symmetric functions associated with Coxeter groups, when it became apparent that this investigation involved fundamental combinatorial structures of independent interest.

In retrospect, perhaps the best way to introduce this subject is as the completion of an analogy. One of the motivations guiding the development of Stanley's theory of (ordinary) $P$-partitions ${ }^{1}$ has been the combinatorics of semistandard tableaux associated with Schur's $S$-functions. Indeed, an (ordinary) $P$-partition can be viewed as a type of generalized tableau - a mapping that assigns entries to the elements of a poset - with various rules for specifying when equality of adjacent entries is allowed. On the other hand, although it did not exist at the time of Stanley's monograph [St1], there is a combinatorial theory for Schur's $Q$-functions that parallels in many ways the corresponding theory for Schur's $S$-functions. In particular, Schur's $Q$-functions are generating functions for a type of tableau attached to shifted Young diagrams, the entries being subjected to a different set of rules than those used for Schur's $S$-functions. Enriched $P$-partitions are the generalized "tableaux" one obtains by keeping these new rules, but replacing the shifted Young diagrams with arbitrary partially ordered sets.

Almost every aspect of the theory of ordinary $P$-partitions has an enriched counterpart. For example, in the ordinary theory, the descent set $\left\{i: w_{i}>w_{i+1}\right\}$ of

Received by the editors August 25, 1994.

1991 Mathematics Subject Classification. Primary 06A07, 05E05 .

Partially supported by NSF Grants DMS-9057192 and DMS-9401575 .

${ }^{1}$ It would be better to refer to these as "poset partitions," but we instead follow tradition. 
a linear extension $w$ plays a central rôle. In the enriched case, this rôle is played by the peak set; i.e., the set $\left\{i: w_{i-1}<w_{i}>w_{i+1}\right\}$. As another example, in the ordinary theory, the order polynomial counts the number of $P$-partitions with entries $\leq m$, and there is a reciprocity theorem relating the order polynomial of a poset to the order polynomial of a complementary poset. In the enriched case, there is a self-reciprocity theorem.

\section{Ordinary P-Partitions}

We begin by reviewing some salient parts of the theory of ordinary $P$-partitions, as developed by Stanley in [St1]. (See also [St2, §4.5].) It should be noted that in [St1], $P$-partitions are required to be order-reversing maps, whereas here they are order-preserving. This does not affect the theory in any substantive way.

All posets considered in this paper will be finite.

1.1 Labeled posets. By a labeled poset, we shall mean a pair $(P, \gamma)$, where $P=$ $(X, \leq)$ is a partial ordering of a (finite) set $X$, and $\gamma: X \rightarrow A$ is an injective map that assigns labels to the elements of $X$ from a totally ordered alphabet $A$. For convenience, we will often assume that $X=[n]=\{1,2, \ldots, n\}$ for some $n$. One says that the labeling $\gamma$ is natural if $x<y$ implies $\gamma(x)<\gamma(y)$ for all $x, y \in X$. The labeling dual to $\gamma$, denoted $\gamma^{*}$, is obtained by reversing the total order on $A$. Also, the poset dual to $P$, namely $(X, \geq)$, is denoted $P^{*}$.

Let $\mathbf{P}$ denote the set of positive integers. A $(P, \gamma)$-partition is a map $f: X \rightarrow \mathbf{P}$ such that for all $x<y$ in $P$, we have

(i) $f(x) \leq f(y)$,

(ii) $f(x)<f(y)$ whenever $\gamma(x)>\gamma(y)$.

We remark that it suffices to impose conditions (i) and (ii) when $x \lessdot y$ (i.e., when $y$ covers $x$, meaning that $x<y$, and $x<z \leq y$ implies $z=y$ ). Note also that if $\gamma$ is natural, then a $(P, \gamma)$-partition is merely an order-preserving map $P \rightarrow \mathbf{P}$. We let $\mathcal{A}(P, \gamma)$ denote the set of $(P, \gamma)$-partitions.

Two labelings $\gamma$ and $\gamma^{\prime}$ of $P$ are defined to be equivalent if $\mathcal{A}(P, \gamma)=\mathcal{A}\left(P, \gamma^{\prime}\right)$. This amounts to having

$$
\gamma(x)>\gamma(y) \Leftrightarrow \gamma^{\prime}(x)>\gamma^{\prime}(y)
$$

for all $x \lessdot y$. This induces a notion of isomorphism for labeled posets: one has $(P, \gamma) \cong(Q, \delta)$ if there is a poset isomorphism $\varphi: P \rightarrow Q$ such that $\delta \varphi$ and $\gamma$ are equivalent labelings of $P$.

Let $H$ denote the ordinary graph induced by the covering relation of $P$, i.e., the graph with vertex set $X$ and edges $\{x, y\}$ for every $x, y \in X$ such that $x \lessdot y$ or $x \gg y$. There is a one-to-one correspondence between equivalence classes of labelings of $P$ and acyclic orientations of $H$. The labeling $\gamma$ corresponds to the orientation $\vec{H}$ in which the edge $\{x, y\}$ is oriented $x \rightarrow y$ if and only if $\gamma(x)>\gamma(y)$. The orientation of $H$ corresponding to the equivalence class of natural labelings can be identified with the Hasse diagram of $P$.

1.2 Generating functions. Let $z_{1}, z_{2}, \ldots$ be commuting indeterminates. We define the weight enumerator of $(P, \gamma)$ to be the formal power series

$$
\Gamma(P, \gamma):=\sum_{f \in \mathcal{A}(P, \gamma)} \prod_{x \in X} z_{f(x)} .
$$


We remark that $\Gamma(P, \gamma)$ is obviously quasi-symmetric; i.e., for each fixed tuple of integers $\left(a_{1}, \ldots, a_{l}\right)$, the coefficient of the monomial $z_{i_{1}}^{a_{1}} \cdots z_{i_{l}}^{a_{l}}$ is constant for $i_{1}<\cdots<i_{l}$.

If $f: X \rightarrow[m]$ is a $(P, \gamma)$-partition with parts $\leq m$, then $f^{*}$ is a $\left(P^{*}, \gamma^{*}\right)$ partition, where $f^{*}(x):=m+1-f(x)$. We therefore have

$$
\Gamma\left(P^{*}, \gamma^{*}\right)\left(z_{1}, \ldots, z_{m}\right)=\Gamma(P, \gamma)\left(z_{m}, \ldots, z_{1}\right) .
$$

Assume $|X|=n$. By a linear extension of $P$, we mean a total ordering $w=$ $\left(w_{1}, \ldots, w_{n}\right)$ of $X$ such that $w_{i}<w_{j}$ in $P$ implies $i<j$. We let $\mathcal{L}(P)$ denote the set of linear extensions of $P$, and we let $D(w, \gamma)=\left\{i: \gamma\left(w_{i}\right)>\gamma\left(w_{i+1}\right)\right\}$ denote the descent set of $w$, relative to the labeling $\gamma$. We will also use the abbreviation $D(w)$ when the labeling is understood.

Any total ordering $w=\left(w_{1}, \ldots, w_{n}\right)$ of the elements of $X$ is in particular a poset, and hence the pair $(w, \gamma)$ forms a labeled poset. Furthermore, the structure of the set of $(w, \gamma)$-partitions is especially simple; indeed,

$$
\mathcal{A}(w, \gamma)=\left\{f: X \rightarrow \mathbf{P} \mid f\left(w_{1}\right) \leq \cdots \leq f\left(w_{n}\right), i \in D(w, \gamma) \Rightarrow f\left(w_{i}\right)<f\left(w_{i+1}\right)\right\} .
$$

In the general case, the Fundamental Lemma on $(P, \gamma)$-Partitions (FLPP) (Theorem 6.2 of [St1]) asserts that the set of $(P, \gamma)$-partitions is the disjoint union

$$
\mathcal{A}(P, \gamma)=\bigcup_{w \in \mathcal{L}(P)} \mathcal{A}(w, \gamma)
$$

Thus as a corollary, we have

$$
\Gamma(P, \gamma)=\sum_{w \in \mathcal{L}(P)} \Gamma(w, \gamma)
$$

A number of specializations of $\Gamma(P, \gamma)$ have combinatorial significance. For example, if we take $\left(z_{1}, z_{2}, \ldots\right)=\left(q, q^{2}, q^{3}, \ldots\right)$, we obtain the generating function for $(P, \gamma)$-partitions $f$ according to the sum of the parts of $f$. In particular, it is not hard to show that $\Gamma(w, \gamma)\left(q, q^{2}, \ldots\right)=q^{\operatorname{ind}(w, \gamma)} /(q)_{n}$, where $(q)_{n}=$ $(1-q)\left(1-q^{2}\right) \cdots\left(1-q^{n}\right)$ and

$$
\operatorname{ind}(w, \gamma)=n+\sum_{i \in D(w, \gamma)} n-i .
$$

Thus by (1.4), we have (Corollaries 5.3 and 7.2 of [St1])

$$
\Gamma(P, \gamma)\left(q, q^{2}, \ldots\right)=\frac{1}{(q)_{n}} \sum_{w \in \mathcal{L}(P)} q^{\operatorname{ind}(w, \gamma)} .
$$

A second important specialization is obtained by taking $\left(z_{1}, z_{2}, \ldots\right)=\left(1^{m}\right)$; that is, $z_{1}=\cdots=z_{m}=1$, and $z_{i}=0$ for $i>m$. In this case,

$$
\Omega(P, \gamma)(m):=\Gamma(P, \gamma)\left(1^{m}\right)
$$

is the number of $(P, \gamma)$-partitions with parts $\leq m$; i.e., the order polynomial of $(P, \gamma)$. It is not hard to show that

$$
\Omega(w, \gamma)(m)=\left(\begin{array}{c}
m+n-d-1 \\
n
\end{array}\right)
$$




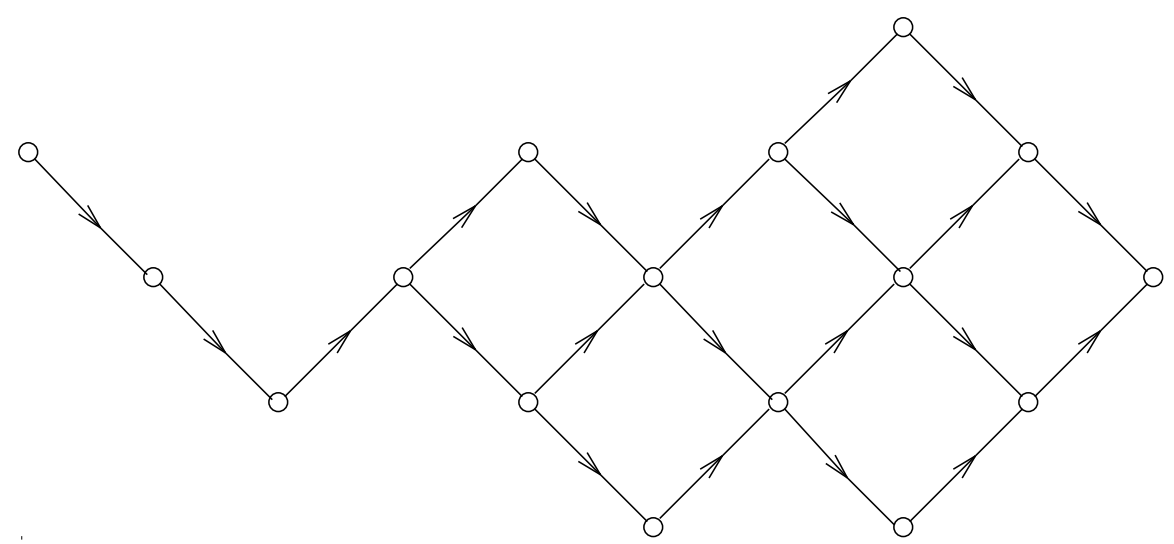

FiguRE 1. An oriented skew diagram.

where $d=|D(w, \gamma)|$, so a further corollary of (1.4) is (Proposition 13.3 of [St1])

$$
\sum_{m \geq 0} \Omega(P, \gamma)(m) t^{m}=\frac{1}{(1-t)^{n+1}} \sum_{w \in \mathcal{L}(P)} t^{1+|D(w, \gamma)|}
$$

1.3 Skew shapes. One of the primary examples that motivates the definition of $(P, \gamma)$-partitions are the labeled posets associated with Young diagrams and the corresponding theory of Schur functions. Given a pair of partitions, say $\lambda=$ $\left(\lambda_{1} \geq \lambda_{2} \geq \cdots\right)$ and $\mu=\left(\mu_{1} \geq \mu_{2} \geq \cdots\right)$, satisfying $\lambda_{i} \geq \mu_{i}$, the corresponding (skew) diagram is

$$
D_{\lambda / \mu}=\left\{(i, j) \in \mathbf{Z}^{2}: i \geq 1, \mu_{i}<j \leq \lambda_{i}\right\},
$$

partially ordered by means of the usual product order on $\mathbf{Z}^{2}$. A labeling $\gamma$ for $D_{\lambda / \mu}$ will be said to be canonical if $\gamma(i, j)<\gamma(i, j+1)$ and $\gamma(i, j)>\gamma(i+1, j)$. (For example, in Figure 1 is the Hasse diagram of $D_{64433 / 31}$, together with the orientation corresponding to a canonical labeling.) In this case, the $\left(D_{\lambda / \mu}, \gamma\right)$-partitions are precisely the semistandard tableaux of shape $\lambda / \mu$, and the weight enumerator of $\left(D_{\lambda / \mu}, \gamma\right)$ is the skew Schur function of shape $\lambda / \mu$; i.e.,

$$
\Gamma\left(D_{\lambda / \mu}, \gamma\right)=s_{\lambda / \mu}
$$

In particular, the weight enumerator of $\left(D_{\lambda / \mu}, \gamma\right)$ is a symmetric function of $z_{1}, z_{2}$, $\ldots$, and not merely quasi-symmetric.

If the labeling of the poset of a skew shape is left unspecified, it will be implicitly assumed to be canonical.

Let $\mathcal{F}^{\lambda / \mu}$ denote the set of standard Young tableaux of shape $\lambda / \mu$, i.e., the set of order-preserving bijections $T: D_{\lambda / \mu} \rightarrow[n]$, where $n$ is the number of elements of $D_{\lambda / \mu}$. (Alternatively, these are the $D_{\lambda / \mu}$-partitions of weight $z_{1} \cdots z_{n}$.) There is a natural identification between $\mathcal{F}^{\lambda / \mu}$ and $\mathcal{L}\left(D_{\lambda / \mu}\right)$. We define the descent set $D(T)$ of a standard tableau $T$ to be the descent set of the corresponding linear extension of $D_{\lambda / \mu}$. Consequently, one has $k \in D(T)$ if and only if $T^{-1}(k)$ occurs to the left (cf. Figure 1) of $T^{-1}(k+1)$ in $D_{\lambda / \mu}$ (i.e., $T(i, j)=k$ and $T\left(i^{\prime}, j^{\prime}\right)=k+1$, where $\left.i<i^{\prime}\right)$. 
1.4 Quasi-symmetric functions. Following Gessel [G], let $\boldsymbol{\Sigma}=\bigoplus_{n \geq 0} \boldsymbol{\Sigma}^{n}$ denote the (graded) ring of quasi-symmetric functions in the variables $z_{1}, z_{2}, \ldots$, with coefficients in $\mathbf{Z}$. For each composition (i.e., ordered sequence of positive integers) $\alpha=\left(\alpha_{1}, \ldots, \alpha_{l}\right)$ with sum $n$, let

$$
M_{\alpha}:=\sum_{i_{1}<\cdots<i_{l}} z_{i_{1}}^{\alpha_{1}} \cdots z_{i_{l}}^{\alpha_{l}}
$$

denote the monomial quasi-symmetric function indexed by $\alpha$. It is clear that as $\alpha$ varies over the compositions of $n$, the $M_{\alpha}$ 's form a free basis for $\boldsymbol{\Sigma}^{n}$ as a $\mathbf{Z}$-module.

In circumstances where the degree $n$ is understood, it is convenient to index the monomial quasi-symmetric functions by subsets of $[n-1]$. That is, for $D=$ $\left\{a_{1}<\cdots<a_{l-1}\right\} \subset[n-1]$, we write $M_{D}$ for $M_{\alpha}$, where $\alpha_{j}=a_{j}-a_{j-1}, a_{0}=0$, and $a_{l}=n$. We also define

$$
L_{D}=\sum_{D \subset E \subset[n-1]} M_{E}=\sum_{\substack{i_{1} \leq \cdots \leq i_{n} \\ k \in D \Rightarrow i_{k}<i_{k+1}}} z_{i_{1}} \cdots z_{i_{n}} .
$$

Since the transition matrix between the $L_{D}$ 's and $M_{D}$ 's is unitriangular, it follows that the $L_{D}$ 's form another free basis for $\boldsymbol{\Sigma}^{n}$.

Comparing the definition of $L_{D}$ with (1.3), we see that $\Gamma(w, \gamma)=L_{D(w, \gamma)}$. Hence (1.4) can be rewritten in the form

$$
\Gamma(P, \gamma)=\sum_{w \in \mathcal{L}(P)} L_{D(w, \gamma)}
$$

In the special case of a skew shape $\lambda / \mu$, this becomes

$$
s_{\lambda / \mu}=\Gamma\left(D_{\lambda / \mu}\right)=\sum_{T \in \mathcal{F}^{\lambda / \mu}} L_{D(T)} .
$$

Although it is possible to verify directly that the quasi-symmetric functions do form a ring, this fact is more transparent from the point of view of $(P, \gamma)$-partitions. Indeed, it is immediate from the definition of the weight enumerator that

$$
\Gamma(P, \gamma) \Gamma(Q, \delta)=\Gamma((P, \gamma)+(Q, \delta)),
$$

where '+' denotes disjoint union of labeled posets. Since $\Gamma(w, \gamma)=L_{D(w, \gamma)}$, one knows that the weight enumerators of labeled posets span all of $\boldsymbol{\Sigma}$; this proves the assertion.

From the previous observation, one can also determine the structure constants of $\boldsymbol{\Sigma}$ relative to the basis of $L_{D}$ 's. First, choose $L_{D} \in \boldsymbol{\Sigma}^{m}$ (hence $D \subset[m-1]$ ) and $L_{E} \in \boldsymbol{\Sigma}^{n}$ (hence $E \subset[n-1]$ ), and arbitrarily partition $[m+n]$ into disjoint sets $X$ and $Y$ of sizes $m$ and $n$, respectively. Second, choose labeled total orders $(u, \gamma)$ and $(v, \delta)$ of $X$ and $Y$ so that $D(u, \gamma)=D$ and $D(v, \delta)=E$. Under these circumstances, we have

$$
L_{D} \cdot L_{E}=\Gamma((u, \delta)+(v, \gamma))=\sum_{w \in \mathcal{S}(u, v)} L_{D(w, \delta \cup \gamma)},
$$

where $\mathcal{S}(u, v)$ denotes the set of linear extensions of the disjoint union of $u$ and $v$ (i.e., the set of shuffles of $u$ and $v$ ). 


\section{ENRICHED $P$-PARTITIONS}

Let $\mathbf{P}^{\prime}$ denote the set of nonzero integers, totally ordered so that

$$
-1 \prec+1 \prec-2 \prec+2 \prec-3 \prec+3 \prec \cdots .
$$

For $k \in \mathbf{P}^{\prime}$, the notations $k>0$ and $|k|$ retain their usual meaning.

Let $(P, \gamma)$ be a labeled poset with vertex set $X$. An enriched $(P, \gamma)$-partition is a map $f: P \rightarrow \mathbf{P}^{\prime}$ such that for all $x<y$ in $P$, we have

(i) $f(x) \preccurlyeq f(y)$,

(ii) $f(x)=f(y)>0$ implies $\gamma(x)<\gamma(y)$,

(iii) $f(x)=f(y)<0$ implies $\gamma(x)>\gamma(y)$.

As in the ordinary case, it suffices to impose conditions (i)-(iii) only when $y$ covers $x$. We let $\mathcal{E}(P, \gamma)$ denote the set of enriched $(P, \gamma)$-partitions.

2.1 The fundamental lemma. Let $w=\left(w_{1}, \ldots, w_{n}\right)$ be a total ordering of the elements of $X$. As in the ordinary case, the structure of the set of enriched $(w, \gamma)$ partitions is quite simple; one has (cf. (1.3))

$$
\begin{aligned}
\mathcal{E}(w, \gamma)=\{f: X \rightarrow \mathbf{P} \mid & f\left(w_{1}\right) \preccurlyeq \cdots \preccurlyeq f\left(w_{n}\right), \\
f\left(w_{i}\right) & =f\left(w_{i+1}\right)>0 \Rightarrow i \notin D(w, \gamma), \\
f\left(w_{i}\right) & \left.=f\left(w_{i+1}\right)<0 \Rightarrow i \in D(w, \gamma)\right\} .
\end{aligned}
$$

The following is the Fundamental Lemma on Enriched $(P, \gamma)$-Partitions (FLEPP).

Lemma 2.1. For any labeled poset $(P, \gamma)$, we have

$$
\mathcal{E}(P, \gamma)=\bigcup_{w \in \mathcal{L}(P)} \mathcal{E}(w, \gamma)
$$

Proof. Given an enriched $(P, \gamma)$-partition $f$, arrange the elements of $X$ in increasing order (relative to $\prec$ ) of their $f$-values, breaking ties as follows: if there are several elements $x$ of $X$ such that $f(x)=-k<0$ (resp., $f(x)=+k>0$ ), arrange them in order of decreasing (resp., increasing) values of $\gamma$. The resulting ordering forms a linear extension $w$ of $P$ such that $f \in \mathcal{E}(w, \gamma)$, and it is not hard to see that $w$ is the unique such extension. Since the members of $\mathcal{E}(w, \gamma)$ are indeed enriched $(P, \gamma)$-partitions, the result follows.

For integers $k>0$, we assign the weight $z_{k}$ to both $k$ and $-k$ (as members of $\left.\mathbf{P}^{\prime}\right)$, so that the weight enumerator for enriched $(P, \gamma)$-partitions is the formal power series

$$
\Delta(P, \gamma):=\sum_{f \in \mathcal{E}(P, \gamma)} \prod_{x \in X} z_{|f(x)|} \cdot
$$

It is clear that $\Delta(P, \gamma)$ is a homogeneous quasi-symmetric function, and an immediate consequence of the FLEPP is the expansion

$$
\Delta(P, \gamma)=\sum_{w \in \mathcal{L}(P)} \Delta(w, \gamma)
$$

Also, in contrast to (1.2), we have

$$
\Delta\left(P^{*}, \gamma\right)\left(z_{1}, \ldots, z_{m}\right)=\Delta(P, \gamma)\left(z_{m}, \ldots, z_{1}\right),
$$

since reversing the chain $-1 \prec+1 \prec \cdots \prec-m \prec+m$ also reverses signs. 
2.2 Peak sets. It is clear from the definition that $\Delta(w, \gamma)$ depends only on the descent set $D(w, \gamma)$; more generally, $\Delta(P, \gamma)$ depends only on the distribution of descents in $\mathcal{L}(P)$, relative to $\gamma$. The same is also true for the weight enumerators of ordinary $(P, \gamma)$-partitions. It is less clear, but nevertheless true (we claim), that $\Delta(w, \gamma)$ depends only on the peak set of $w$ relative to $\gamma$, i.e., the set

$$
\Lambda(w, \gamma):=\left\{i: 1<i<n, \gamma\left(w_{i-1}\right)<\gamma\left(w_{i}\right)>\gamma\left(w_{i+1}\right)\right\} .
$$

This claim is a consequence of the following.

Proposition 2.2. We have

$$
\Delta(w, \gamma)=\sum_{E \subset[n-1]: \Lambda(w, \gamma) \subset E \cup(E+1)} 2^{|E|+1} M_{E} .
$$

Proof. Choose $E=\left\{a_{1}<\cdots<a_{l-1}\right\} \subset[n-1]$. The coefficient of $M_{E}$ in $\Delta(w, \gamma)$ is also the coefficient of $z_{1}^{\alpha_{1}} \cdots z_{l}^{\alpha_{l}}$ in $\Delta(w, \gamma)$, where $\alpha_{j}=a_{j}-a_{j-1}, a_{0}=0$, and $a_{l}=n$. The only enriched $(w, \gamma)$-partitions $f$ with this weight must satisfy

$$
\left(\left|f\left(w_{1}\right)\right|, \ldots,\left|f\left(w_{n}\right)\right|\right)=(\underbrace{1, \ldots, 1}_{\alpha_{1}}, \underbrace{2, \ldots, 2}_{\alpha_{2}}, \ldots, \underbrace{l, \ldots, l}_{\alpha_{l}}),
$$

so the only choices to be resolved are the signs of the $f\left(w_{i}\right)$ 's. However, $f\left(w_{1}\right), \ldots$, $f\left(w_{n}\right)$ must be non-decreasing relative to $\prec$, so every -1 must precede every occurrence of +1 , and so on. Hence, the only choices to resolve are the number of occurrences of $-k$ in $f$ for $1 \leq k \leq l$. From (2.1) we see that the possible choices are characterized by the fact that for each $k$, the subsequence of $w$ whose $f$-values are $-k$ (resp., $+k$ ) must be decreasing (resp., increasing) relative to $\gamma$. In particular, the subsequence of $w$ whose $f$-values are $\pm k$ must be free of peaks, relative to $\gamma$. Conversely, given a peak-free subsequence of $w$, there are exactly two ways to assign $\pm k$ to the terms of this subsequence so as to meet the condition: if $w_{i}$ is the unique term that minimizes $\gamma$ in this subsequence, then $f\left(w_{i}\right)$ can be assigned $\pm k$ arbitrarily; all terms preceding (resp., succeeding) $w_{i}$ must be assigned $-k$ (resp., $+k)$ by $f$.

From this analysis, we see that the coefficient of $M_{E}$ in $\Delta(w, \gamma)$ is nonzero if and only if the partition of $w$ into subsequences induced by $E$ is free of peaks, relative to $\gamma$. In that case, the coefficient of $M_{E}$ is $2^{|E|+1}$, since $|E|+1$ is the number of such subsequences. Finally, observe that $E$ induces a peak-free partition of $w$ if and only if $j \in E$ or $j \in E+1$ for every $j \in \Lambda(w, \gamma)$.

Any subset $\Lambda \subset[n]$ is said to be a peak set if $\Lambda=\Lambda(w, \gamma)$ for some $(w, \gamma)$; i.e., $i \in \Lambda \Rightarrow i \pm 1 \notin \Lambda$, and $1, n \notin \Lambda$. For $n>0$, the number of peak sets in $[n]$ is the Fibonacci number $f_{n}$ (indexed so that $f_{1}=f_{2}=1$ ).

The above result suggests we define a family of quasi-symmetric functions $K_{\Lambda} \in$ $\boldsymbol{\Sigma}^{n}$ indexed by peak sets, by setting

$$
K_{\Lambda}:=\Delta(w, \gamma)
$$

for any $(w, \gamma)$ such that $\Lambda=\Lambda(w, \gamma)$. In these terms, (2.2) can be rewritten in the form

$$
\Delta(P, \gamma)=\sum_{w \in \mathcal{L}(P)} K_{\Lambda(w, \gamma)}
$$




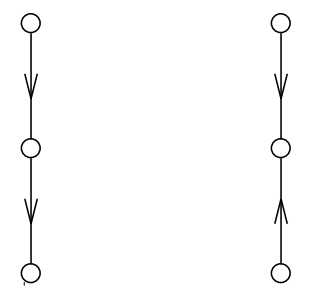

FiguRE 2. Weak equivalence.

2.3 Weak equivalence. For the study of enriched $(P, \gamma)$-partitions, the notion of equivalence of labelings (see Section 1.1) is too strong. For example, consider the two (inequivalent) labelings $\gamma_{L}$ and $\gamma_{R}$ of the 3-element chain in Figure 2. (Here we follow our convention of depicting the labeling of a poset by means of an orientation of the Hasse diagram.) If $f \in \mathcal{E}\left([3], \gamma_{L}\right)$ satisfies $f(1) \prec f(2)$, then $f \in \mathcal{E}\left([3], \gamma_{R}\right)$, and the same is true with $L$ and $R$ interchanged. Otherwise, if $f(1)=f(2)$ and we define $g(1)=-f(1), g(2)=-f(2), g(3)=f(3)$, then $g \in \mathcal{E}\left([3], \gamma_{R}\right)$, and again the same is true with $L$ and $R$ interchanged. Thus there is a bijection $f \mapsto f^{\prime}$ from $\mathcal{E}\left([3], \gamma_{L}\right)$ to $\mathcal{E}\left([3], \gamma_{R}\right)$ such that $|f(i)|=\left|f^{\prime}(i)\right|$ for $1 \leq i \leq 3$. We propose that it is therefore reasonable to regard these labelings as "equivalent."

In the general case, let $\|\mathcal{E}(P, \gamma)\|$ denote the multiset $\{|f|: f \in \mathcal{E}(P, \gamma)\}$. (We use the notation $\|\cdot\|$ here to avoid confusion with the interpretation of $|\cdot|$ as the cardinality function for sets.) We define two labelings $\gamma$ and $\gamma^{\prime}$ of $P$ to be weakly equivalent if $\|\mathcal{E}(P, \gamma)\|=\left\|\mathcal{E}\left(P, \gamma^{\prime}\right)\right\|$. Similarly, two labeled posets $(P, \gamma)$ and $(Q, \delta)$ are said to be weakly isomorphic, written $(P, \gamma) \approx(Q, \delta)$, if there is a poset isomorphism $\varphi: P \rightarrow Q$ such that $\delta \varphi$ and $\gamma$ are weakly equivalent labelings of $P$. It is clear that $\Delta(P, \gamma)$ depends only on the weak isomorphism class of $(P, \gamma)$.

A subposet $(Y, \leq)$ of $P$ is said to be convex if $x<y<z$ in $P$ and $x, z \in Y$ implies $y \in Y$.

Proposition 2.3. If $\gamma$ and $\gamma^{\prime}$ are weakly equivalent labelings of $P$, then for every convex subposet of $P$, the labelings induced by $\gamma$ and $\gamma^{\prime}$ are also weakly equivalent.

Proof. Let $Q=(Y, \leq)$ be a convex subposet of $P$, and let $w$ be a linear extension of $P$ such that $Y=\left\{w_{i+1}, \ldots, w_{j}\right\}$. Choose an order-preserving map $g: Y \rightarrow \mathbf{P}$, let $m$ be the maximum value of $g$ on $Y$, and define $\hat{g}: X \rightarrow \mathbf{P}$ by setting

$$
\hat{g}\left(w_{r}\right)=\left\{\begin{array}{cl}
r & \text { if } r \leq i, \\
g\left(w_{r}\right)+i & \text { if } i<r \leq j, \\
m+r & \text { if } r>j .
\end{array}\right.
$$

A map $\hat{f}: X \rightarrow \mathbf{P}^{\prime}$ such that $|\hat{f}|=\hat{g}$ will be an enriched $(P, \gamma)$-partition if and only if the restriction of $\hat{f}$ to $Y$ is an enriched $(Q, \gamma)$-partition. Since the choice of signs for $\hat{f}(x)= \pm \hat{g}(x)$ can be made independently and arbitrarily for $x \notin Y$, it follows that the multiplicity of $\hat{g}$ in $\|\mathcal{E}(P, \gamma)\|$ is $2^{n-|Y|}$ times the multiplicity of $g$ in $\|\mathcal{E}(Q, \gamma)\|$. Hence $\|\mathcal{E}(P, \gamma)\|$, which determines the weak isomorphism class of $\gamma$ as a labeling of $P$, also determines $\|\mathcal{E}(Q, \gamma)\|$.

To give a criterion for testing weak equivalence, ${ }^{2}$ consider the set $J(P)$ of order ideals of $P$, that is, the set of subsets $I \subset X$ satisfying $x \in I, y<x \Rightarrow y \in I$.

\footnotetext{
${ }^{2}$ Unfortunately, there is no criterion for weak equivalence as simple as (1.1).
} 

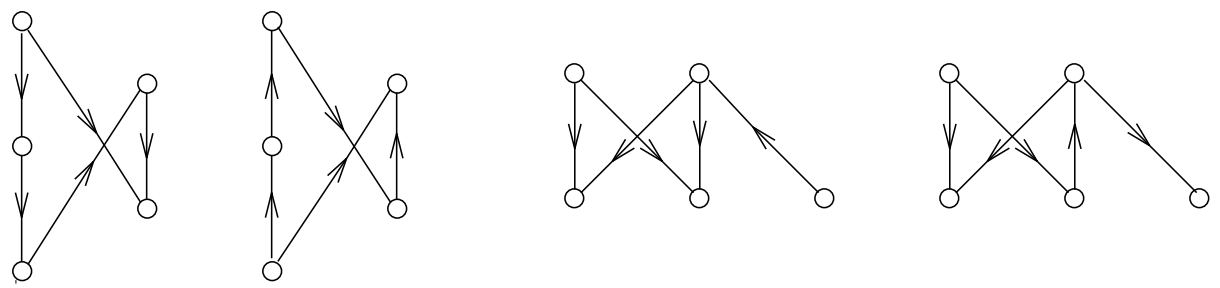

Figure 3. $\Delta(P, \gamma)=\Delta\left(P, \gamma^{\prime}\right) \nRightarrow(P, \gamma) \approx\left(P, \gamma^{\prime}\right)$.

Given a labeling $\gamma$ of $P$, we define binary relations $\rightarrow$ and $\leftarrow$ on $J(P)$ so that

$$
\begin{array}{ll}
I \rightarrow J & \text { if } I \subset J, \text { and } x, y \in J-I, x<y \Rightarrow \gamma(x)>\gamma(y), \\
I \leftarrow J & \text { if } I \subset J, \text { and } x, y \in J-I, x<y \Rightarrow \gamma(x)<\gamma(y) .
\end{array}
$$

Finally, for any $I \subset J$, we define

$$
N(P, \gamma)(I, J):=|\{K \in J(P): I \rightarrow K \leftarrow J\}|,
$$

an element of the incidence algebra of $J(P)$.

We remark that $(Y, \leq)$ is a convex subposet of $P$ if and only if $Y=J-I$ for some pair of order ideals $I \subset J$ of $P$.

Proposition 2.4. If $\gamma$ and $\gamma^{\prime}$ are labelings of a poset $P$, the following are equivalent.

(a) $\gamma$ and $\gamma^{\prime}$ are weakly equivalent.

(b) $\Delta(Q, \gamma)=\Delta\left(Q, \gamma^{\prime}\right)$ for every convex subposet $Q$ of $P$.

(c) $N(P, \gamma)=N\left(P, \gamma^{\prime}\right)$.

Proof. The fact that (a) implies (b) is a consequence of Proposition 2.3.

For any pair of order ideals $I \subset J$ of $P$, we have

$$
N(P, \gamma)(I, J)=\Delta(J-I, \gamma)(1,0,0, \ldots),
$$

which proves that (b) implies (c).

Finally, given any order-preserving map $g: X \rightarrow \mathbf{P}$, let $I_{j}=\{x \in X: g(x) \leq j\}$. The multiplicity of $g$ in $\|\mathcal{E}(P, \gamma)\|$ is $N\left(\varnothing, I_{1}\right) N\left(I_{1}, I_{2}\right) N\left(I_{2}, I_{3}\right) \cdots$, where $N=$ $N(P, \gamma)$. Hence $\|\mathcal{E}(P, \gamma)\|$ is determined by $N(P, \gamma)$, which proves that (c) implies (a).

We note that $\Delta(P, \gamma)=\Delta\left(P, \gamma^{\prime}\right)$ does not imply that $\gamma$ and $\gamma^{\prime}$ are weakly equivalent. Indeed, among the posets with $\leq 5$ vertices, there are three posets that have a pair of non weak-equivalent labelings with the same weight enumerator. In Figure 3 are the orientations for two of these pairs, the third being the dual of the pair on the right.

2.4 Shifted skew shapes. One of the primary motivations for the study of enriched $(P, \gamma)$-partitions are the labeled posets associated with shifted skew shapes and the corresponding theory of Schur's $Q$-functions. (For more on the latter, see the Appendix.) Given a pair of strict partitions, say $\lambda=\left(\lambda_{1}>\lambda_{2}>\cdots\right)$ and $\mu=\left(\mu_{1}>\mu_{2}>\cdots\right)$, satisfying $\lambda_{i} \geq \mu_{i}$, the corresponding shifted skew diagram is

$$
D_{\lambda / \mu}^{\prime}=\left\{(i, j) \in \mathbf{Z}^{2}: i \geq 1, \mu_{i}+i \leq j<\lambda_{i}+i\right\},
$$




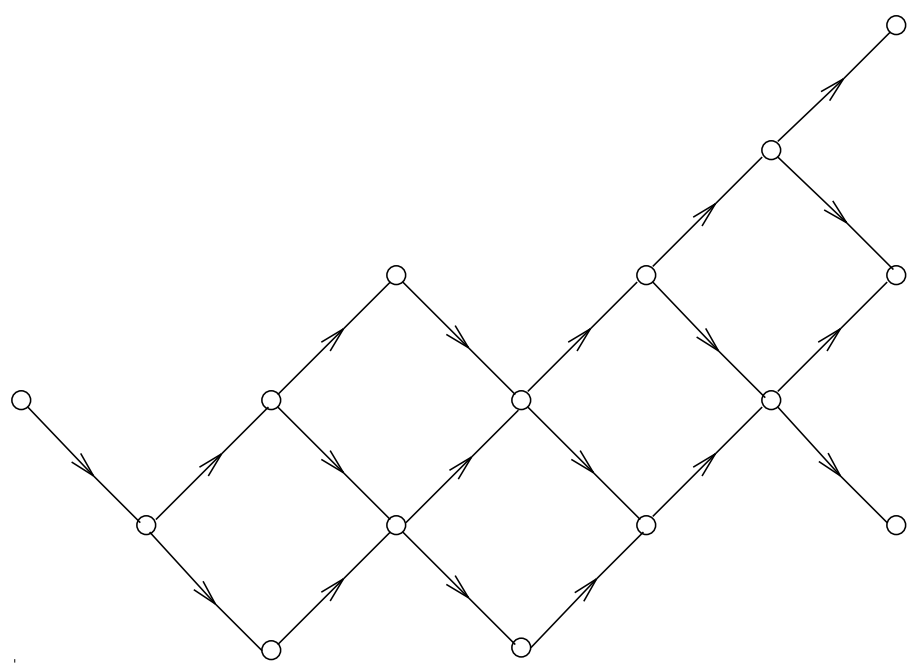

Figure 4. An oriented shifted skew diagram.

partially ordered by means of the usual product order on $\mathbf{Z}^{2}$. We remark that these posets can be identified as the (finite) convex subsets of $\left\{(i, j) \in \mathbf{Z}^{2}: 1 \leq i \leq j\right\}$. Also, every ordinary skew diagram (see Section 1.3) is order-isomorphic to a shifted skew diagram, but not conversely.

As with ordinary skew diagrams, we define a labeling $\gamma$ of $D_{\lambda / \mu}^{\prime}$ to be canonical if $\gamma(i, j)<\gamma(i, j+1)$ and $\gamma(i, j)>\gamma(i+1, j)$; if unspecified, the labeling of a shifted skew diagram will always be assumed to be canonical. For example, in Figure 4 is the oriented Hasse diagram corresponding to a canonical labeling of $D_{865321 / 532}^{\prime}$. It is possible for a given shifted skew diagram to have many inequivalent labelings that are weak-equivalent to the canonical labeling. For example, the poset $D_{43 / 1}^{\prime} \cong$ $D_{33 / \emptyset}$ has 16 such labelings, whereas the poset $D_{421 / \emptyset}^{\prime}$ has only one.

Relative to the canonical labeling, the enriched $D_{\lambda / \mu}^{\prime}$-partitions are also known as the shifted $\mathbf{P}^{\prime}$-tableaux of shape $\lambda / \mu$; their weight enumerator is precisely the skew Schur $Q$-function of shape $\lambda / \mu$ (see Section A.1). Thus we have

$$
\Delta\left(D_{\lambda / \mu}^{\prime}\right)=Q_{\lambda / \mu}
$$

Since Schur's $Q$-functions are in fact symmetric in the variables $z_{1}, z_{2}, \ldots,{ }^{3}$ we see in particular that the enriched weight enumerators for canonically labeled shifted skew diagrams are symmetric, and not merely quasi-symmetric. On the other hand, $\Gamma\left(D_{\lambda / \mu}^{\prime}\right)$ need not be symmetric.

Let $\mathcal{G}^{\lambda / \mu}$ denote the set of shifted standard tableaux of shape $\lambda / \mu$, i.e., the set of order-preserving bijections $T: D_{\lambda / \mu}^{\prime} \rightarrow[n]$, where $n$ is the number of elements of $D_{\lambda / \mu}^{\prime}$. As in the ordinary case, there is a natural identification between $\mathcal{G}^{\lambda / \mu}$ and $\mathcal{L}\left(D_{\lambda / \mu}^{\prime}\right)$. For $T \in \mathcal{G}^{\lambda / \mu}$, we define the descent set $D(T)$ and peak set $\Lambda(T)$ so as to agree with the descent and peak sets of the corresponding $w \in \mathcal{L}\left(D_{\lambda / \mu}^{\prime}\right)$. Thus $k \in D(T)$ if and only if $T^{-1}(k)$ occurs to the left of $T^{-1}(k+1)$, and $\Lambda(T)=$ $\S 6]$.

${ }^{3}$ For a direct proof of this fact, expressible in the language of enriched $P$-partitions, see [Ste2, 
$\{k \in D(T): k-1 \notin D(T)\}$. By (2.4), the enriched counterpart of (1.8) is the expansion

$$
Q_{\lambda / \mu}=\Delta\left(D_{\lambda / \mu}^{\prime}\right)=\sum_{T \in \mathcal{G}^{\lambda / \mu}} K_{\Lambda(T)} .
$$

\section{The ALGEBRA of PEAKS}

For integers $n \geq 0$, let $\boldsymbol{\Pi}^{n}$ denote the $\mathbf{Z}$-submodule of $\boldsymbol{\Sigma}^{n}$ generated by the quasi-symmetric functions $K_{\Lambda}$, where $\Lambda$ ranges over the peak sets in [n], and set $\boldsymbol{\Pi}:=\bigoplus_{n \geq 0} \boldsymbol{\Pi}^{n}$. Note that $\Delta(P, \gamma) \in \boldsymbol{\Pi}$ for every labeled poset $(P, \gamma)$, by (2.4).

Theorem 3.1. $\Pi$ is a graded subring of $\boldsymbol{\Sigma}$. Moreover,

(a) For $n \geq 0, \Pi^{n}$ is freely generated as a $\mathbf{Z}$-module by the $K_{\Lambda}$ 's.

(b) For $n>0$, the rank of $\boldsymbol{\Pi}^{n}$ is the Fibonacci number $f_{n}$.

(c) The map $\Gamma(P, \gamma) \mapsto \Delta(P, \gamma)$ is well-defined, and extends (uniquely) to a graded, surjective ring homomorphism $\theta: \boldsymbol{\Sigma} \rightarrow \boldsymbol{\Pi}$.

Proof. Totally order the subsets of $[n]$, first by cardinality, then by decreasing order of the smallest element, then by decreasing order of the second smallest element, and so on. We therefore have

$$
\varnothing<\cdots<\{2\}<\{1\}<\cdots<\{2,3\}<\cdots<\{1,3\}<\{1,2\}<\cdots .
$$

With respect to this ordering, Proposition 2.2 shows that the leading term in the monomial expansion of $K_{\Lambda}$ is $2^{|\Lambda|+1} M_{\Lambda}$, for any peak set $\Lambda \subset[n]$. Hence, the $K_{\Lambda}$ 's are linearly independent over $\mathbf{Q}$, which proves (a). The fact that the number of peak sets in $[n]$ is $f_{n}$ was noted already in Section 2.2.

To prove that $\boldsymbol{\Pi}$ is a (graded) ring, choose $K_{\Lambda_{1}} \in \Pi^{m}$ and $K_{\Lambda_{2}} \in \Pi^{n}$, where $\Lambda_{1}$ and $\Lambda_{2}$ are peak sets for $[m]$ and $[n]$, respectively. If we partition $[m+n]$ arbitrarily into disjoint sets $X$ and $Y$ of sizes $m$ and $n$, we can find labeled total orders $(u, \gamma)$ and $(v, \delta)$ of $X$ and $Y$ so that $\Lambda(u, \gamma)=\Lambda_{1}$ and $\Lambda(v, \delta)=\Lambda_{2}$. As a consequence of (2.4), we have

$$
K_{\Lambda_{1}} \cdot K_{\Lambda_{2}}=\Delta(u, \gamma) \cdot \Delta(v, \delta)=\Delta((u, \gamma)+(v, \delta))=\sum_{w \in \mathcal{S}(u, v)} K_{\Lambda(w, \gamma \cup \delta)},
$$

where $\mathcal{S}(u, v)$ denotes the set of shuffles of $u$ and $v$.

To prove (c), we can define a $\mathbf{Z}$-linear map $\theta: \boldsymbol{\Sigma}^{n} \rightarrow \boldsymbol{\Pi}^{n}$ by setting $\theta\left(L_{D}\right)=$ $K_{\Lambda(D)}$ for each $D \subset[n-1]$, where

$$
\Lambda(D)=\{i \geq 2: i \in D, i-1 \notin D\} .
$$

A comparison of (1.7) and (2.4) shows that $\theta \Gamma(P, \gamma)=\Delta(P, \gamma)$ for every labeled poset $(P, \gamma)$, and a comparison of (1.9) and (3.1) shows that $\theta$ is a ring homomorphism.

Remark 3.2. As discussed in the Appendix, there is a ring homomorphism, also denoted $\theta$, from the ring $\boldsymbol{\Lambda}$ of symmetric functions onto the subring $\boldsymbol{\Omega}$ generated by the $Q$-functions. Thus we have a collection of ring maps

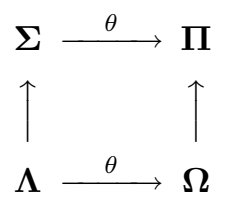


with the vertical maps being inclusions. We claim that this diagram is commutative (so the use of ' $\theta$ ' in both places is consistent). To see this, note that if $([n], \iota)$ is a naturally labeled $n$-element chain, then (using notation from the Appendix) $\Gamma([n], \iota)=h_{n}$ and $\Delta([n], \iota)=q_{n}=\theta\left(h_{n}\right)$. Since the $h_{n}$ 's generate $\boldsymbol{\Lambda}$, this suffices to prove the claim.

A consequence of the above remark and Theorem 3.1 is the following.

Corollary 3.3. If $\Gamma(P, \gamma)$ is symmetric, then so is $\Delta(P, \gamma)$.

Let $\boldsymbol{\Pi}_{\mathbf{Q}}:=\mathbf{Q} \otimes_{\mathbf{Z}} \boldsymbol{\Pi}$ denoted the $\mathbf{Q}$-algebra obtained by replacing the coefficient $\operatorname{ring} \mathbf{Z}$ with the rationals $\mathbf{Q}$. In addition to $\boldsymbol{\Pi}$, there is a second important (graded) subring of $\boldsymbol{\Pi}_{\mathbf{Q}}$ that is free as a $\mathbf{Z}$-module, namely, the ring

$$
\bar{\Pi}:=\Sigma \cap \Pi_{\mathbf{Q}}
$$

consisting of all quasi-symmetric functions with integer coefficients belonging to the $\mathbf{Q}$-span of the $K_{\Lambda}$ 's. The ring $\overline{\boldsymbol{\Pi}}$ bears the same relationship to the ring $\overline{\boldsymbol{\Omega}}$ generated by the Schur $P$-functions as $\Pi$ does to $\boldsymbol{\Omega}$. (See Section A.3.)

For each peak set $\Lambda$ in $[n]$, let us define

$$
\bar{K}_{\Lambda}:=2^{-|\Lambda|-1} K_{\Lambda} .
$$

It is clear from Proposition 2.2 that $\bar{K}_{\Lambda} \in \overline{\boldsymbol{\Pi}}^{n}$.

Corollary 3.4. For $n \geq 0$, the $\bar{K}_{\Lambda}$ 's are a free $\mathbf{Z}$-basis of $\overline{\boldsymbol{\Pi}}^{n}$.

Proof. From the proof of Theorem 3.1, we know that the leading term in the monomial expansion of $\bar{K}_{\Lambda}$ is $M_{\Lambda}$. Hence, the (rectangular) transition matrix between the $\bar{K}_{\Lambda}$ 's and the $M_{E}$ 's is unitriangular and integral. It follows that any rational linear combination of the $\bar{K}_{\Lambda}$ 's in $\boldsymbol{\Sigma}$ must in fact be an integer linear combination.

The following result is a counterpart to Proposition 2.2 ; it provides the $L_{D^{-}}$ expansion of the $K_{\Lambda}$ 's (or equivalently, the $\bar{K}_{\Lambda}$ 's).

Proposition 3.5. For any peak set $\Lambda$ in $[n]$, we have

$$
\bar{K}_{\Lambda}=\sum_{D \subset[n-1]: \Lambda \subset D \Delta(D+1)} L_{D}
$$

where ' $\triangle$ ' denotes symmetric difference; i.e., $D \triangle E=(D-E) \cup(E-D)$.

Proof. Since $L_{D}=\sum_{E \supset D} M_{E}$, the coefficient of $M_{E}$ on the right side of the above expansion is $|\{D \subset E: \Lambda \subset D \triangle(D+1)\}|$. In particular, this number is 0 unless $\Lambda \subset E \cup(E+1)$. In that case, we can count the number of choices for $D$ as follows. For each $j \in \Lambda$ such that both $j, j-1 \in E, D$ must contain exactly one of $j$ or $j-1$; if only one of $j, j-1 \in E$, then that member must also belong to $D$. Any members of $E$ not in $\Lambda \cup(\Lambda-1)$ can be arbitrarily included in $D$. Hence, the number of choices for $D$ is $2^{|E|-|\Lambda|}$, and we have

$$
\sum_{D \subset[n-1]: \Lambda \subset D \Delta(D+1)} L_{D}=\sum_{E \subset[n-1]: \Lambda \subset E \cup(E+1)} 2^{|E|-|\Lambda|} M_{E} .
$$

By Proposition 2.2, this quantity is $2^{-|\Lambda|-1} K_{\Lambda}$. 
In the following, it will be convenient to assume that the alphabet $A$ of labels for partially ordered sets is $\mathbf{Z}$, totally ordered in the usual way. Given any positive labeling $\gamma$ of a poset $P=(X, \leq)$ (i.e., $\gamma: X \rightarrow \mathbf{P}$ ) and a choice of signs $\varepsilon: X \rightarrow$ $\{ \pm 1\}$, let $\varepsilon \gamma$ denote the $P$-labeling $x \mapsto \varepsilon(x) \gamma(x)$. The following result shows that the weight enumerator for enriched $(P, \gamma)$-partitions can be obtained by averaging the weight enumerators of the ordinary $(P, \varepsilon \gamma)$-partitions.

Theorem 3.6. If $\gamma$ is a positive labeling of $P=(X, \leq)$, then

$$
\Delta(P, \gamma)=\sum_{\varepsilon: X \rightarrow\{ \pm 1\}} \Gamma(P, \varepsilon \gamma)
$$

Proof. By the FLEPP and FLPP, it suffices to prove this when $\gamma$ is an arbitrary (positive) labeling of an $n$-element chain $[n]$. For this, let us regard $\varepsilon_{i}=\varepsilon(i)$ $(1 \leq i \leq n)$ as independent, uniformly distributed random variables on $\{ \pm 1\}$, and for $1 \leq j<n$, define $\delta_{j}= \pm 1$ so that $\delta_{j}=+1$ if and only if $j \in D([n], \varepsilon \gamma)$. The random variable $\delta_{j}$ depends only on $\varepsilon_{j}$ or $\varepsilon_{j+1}$; in fact,

$$
\delta_{j}=\left\{\begin{array}{cl}
-\varepsilon_{j+1} & \text { if } \gamma(j)<\gamma(j+1) \\
\varepsilon_{j} & \text { if } \gamma(j)>\gamma(j+1)
\end{array}\right.
$$

It follows that $\delta_{1}, \ldots, \delta_{n-1}$ are uniformly distributed, and that the only pairwise dependencies among them occur between the pairs $\delta_{j-1}$ and $\delta_{j}$ such that $\gamma(j-1)<$ $\gamma(j)>\gamma(j+1)$ (i.e., $j \in \Lambda([n], \gamma)$ ), for which we have $\delta_{j-1}=-\delta_{j}$.

Hence, the only sets $D \subset[n-1]$ of the form $D([n], \varepsilon \gamma)$ for some $\varepsilon$ are those that contain exactly one of $j$ or $j-1$ for each $j \in \Lambda=\Lambda([n], \gamma)$; there are $2^{n-|\Lambda|-1}$ such sets. The independencies among the $\delta_{j}$ 's show that each of these sets $D$ must occur equally often. Since there are $2^{n}$ choices for $\varepsilon$, it follows that each $D$ occurs $2^{|\Lambda|+1}$ times, so we have

$$
\sum_{\varepsilon:[n] \rightarrow\{ \pm 1\}} \Gamma([n], \varepsilon \gamma)=\sum_{\varepsilon:[n] \rightarrow\{ \pm 1\}} L_{D([n], \varepsilon \gamma)}=2^{|\Lambda|+1} \sum_{D \subset[n-1]: \Lambda \subset D \Delta(D+1)} L_{D}
$$

By Proposition 3.5, this quantity is $\Delta([n], \gamma)=K_{\Lambda}$.

Lemma 3.7. Any $f \in \boldsymbol{\Pi}_{\mathbf{Q}}$ satisfies the cancellation law

$$
f\left(t,-t, z_{3}, z_{4}, \ldots\right)=f\left(z_{3}, z_{4}, \ldots\right) \text {. }
$$

Proof. We may assume $f=\Delta(P, \gamma)$ for some labeled poset $(P, \gamma)$. Since

$$
\Delta(P, \gamma)\left(z_{1}, z_{2}, \ldots\right)=\sum_{I \in J(P)} \Delta(I, \gamma)\left(z_{1}, z_{2}\right) \cdot \Delta(P-I, \gamma)\left(z_{3}, z_{4}, \ldots\right)
$$

we see that it suffices to show that $\Delta(P, \gamma)(t,-t)=0$ unless $P$ is the empty poset. For this, it is enough to show that $K_{\Lambda}(t,-t)=0$ for any peak set $\Lambda$ in $[n]$, for $n>0$. Since $M_{D}(s, t)=0$ unless $|D| \leq 1$, it follows from Proposition 2.2 that $K_{\Lambda}(s, t)=0$ unless $|\Lambda| \leq 1$. In case $\Lambda=\{j\}$, Proposition 2.2 also shows that there are only two nonzero terms in the $M_{E}$-expansion of $K_{\Lambda}(s, t)$, yielding $K_{\Lambda}(s, t)=$ $4\left(s^{j} t^{n-j}+s^{j-1} t^{n-j+1}\right)$. Thus it is clear that $K_{\Lambda}(t,-t)=0$. Otherwise, if $\Lambda=\varnothing$, then $K_{\Lambda}=q_{n}$; the fact that $q_{n}$ satisfies the cancellation law is clear from (A.1).

Theorem 3.8. We have $\boldsymbol{\Omega}=\boldsymbol{\Lambda} \cap \boldsymbol{\Pi}$ and $\overline{\boldsymbol{\Omega}}=\boldsymbol{\Lambda} \cap \overline{\boldsymbol{\Pi}}$; i.e., $\boldsymbol{\Omega}$ and $\overline{\boldsymbol{\Omega}}$ are the symmetric parts of $\boldsymbol{\Pi}$ and $\overline{\boldsymbol{\Pi}}$. 
Proof. The fact that $\boldsymbol{\Omega} \subset \boldsymbol{\Pi}$ is clear, since $q_{n}=K_{\varnothing}(n \geq 0)$ are the generators of $\boldsymbol{\Omega}$. Therefore $\boldsymbol{\Omega} \subset \boldsymbol{\Lambda} \cap \boldsymbol{\Pi}$ and hence also $\overline{\boldsymbol{\Omega}} \subset \boldsymbol{\Lambda} \cap \overline{\boldsymbol{\Pi}}$. Conversely, Lemma 3.7 shows that any $f \in \boldsymbol{\Lambda} \cap \overline{\boldsymbol{\Pi}}$ satisfies the cancellation law, and therefore $f \in \overline{\boldsymbol{\Omega}}$, by (A.5). To complete the proof, we must show that $\boldsymbol{\Lambda} \cap \boldsymbol{\Pi} \subset \boldsymbol{\Omega}$. However, since $\boldsymbol{\Lambda} \cap \boldsymbol{\Pi} \subset \boldsymbol{\Lambda} \cap \overline{\boldsymbol{\Pi}}=\overline{\boldsymbol{\Omega}}$, it suffices to show that $\boldsymbol{\Pi} \cap \overline{\boldsymbol{\Omega}} \subset \boldsymbol{\Omega}$.

For this, choose a strict partition $\lambda$ of size $n$ and length $l=\ell(\lambda)$. We claim that every standard shifted tableau $T \in \mathcal{G}^{\lambda}$ must have a peak set of size $\geq l-1$. Indeed, suppose that $T(i, i)=a_{i}(1 \leq i \leq l)$ are the entries on the main diagonal of $T$. For each $i<l$, the cell containing $a_{i}+1$ cannot be in a row numbered greater than $i$ (hence $a_{i} \notin D(T)$ ), and $a_{i+1}-1$ must be in a row numbered $\leq i$ (hence $\left.a_{i+1}-1 \in D(T)\right)$. It follows that for each $i<l$, there must exist some index $j$ $\left(a_{i}<j<a_{i+1}\right)$ such that $j-1 \notin D(T)$ and $j \in D(T)$, or equivalently, $j \in \Lambda(T)$. Hence the claim follows.

Now suppose that $\Lambda(T)=\left\{b_{1}<\cdots<b_{k}\right\}$. Since the subtableau of $T$ formed by the entries $\leq \lambda_{1}+\cdots+\lambda_{i}+1$ must occupy at least $i+1$ rows, the above claim shows that $b_{i} \leq \lambda_{1}+\cdots+\lambda_{i}$. Setting $\Lambda_{0}=\left\{\lambda_{1}+\cdots+\lambda_{i}: 1 \leq i<l\right\}$, it follows that $\Lambda(T) \geq \Lambda_{0}$ for every $T \in \mathcal{G}^{\lambda}$, relative to the total ordering on subsets of $[n]$ introduced in the proof of Theorem 3.1.

There is a unique tableau $T=T_{0} \in \mathcal{G}^{\lambda}$ such that $\Lambda\left(T_{0}\right)=\Lambda_{0}$, namely, the standard tableau corresponding to the linear extension of $D_{\lambda}^{\prime}$ in which every cell in row one precedes every cell in row two, and so on; i.e.,

$$
(1,1)<(1,2)<(1,3)<\cdots<(2,2)<(2,3)<\cdots<(3,3)<(3,4)<\cdots .
$$

Hence, the leading term in the $K_{\Lambda^{-}}$-expansion of $Q_{\lambda}$ (see (2.5)) is $K_{\Lambda_{0}}$. Since distinct $Q_{\lambda}$ 's have distinct leading terms, it follows that the transition matrix is unitriangular. In particular, any integral linear combination of $K_{\Lambda}$ 's (i.e., a member of $\boldsymbol{\Pi}$ ) that is also a rational linear combination of $Q_{\lambda}$ 's (i.e., a member of $\boldsymbol{\Omega}_{\mathbf{Q}}$ ) must in fact be an integer linear combination of $Q_{\lambda}$ 's (i.e., a member of $\boldsymbol{\Omega}$ ).

Corollary 3.9. If $\Delta(P, \gamma)$ is symmetric, then $\Delta(P, \gamma)$ is $Q$-integral, i.e., a $\mathbf{Z}$-linear combination of Schur Q-functions.

\section{THE ENRICHED ORDER POLYNOMIAL}

For a labeled poset $(P, \gamma)$, we define the enriched order polynomial $\Omega^{\prime}(P, \gamma)(t)$ by setting

$$
\Omega^{\prime}(P, \gamma)(m):=\Delta(P, \gamma)\left(1^{m}\right)
$$

for nonnegative integers $m$. Thus $\Omega^{\prime}(P, \gamma)(m)$ is the number of enriched $(P, \gamma)$ partitions with parts $\preccurlyeq m$.

4.1 Basic properties. The fact that $\Omega^{\prime}(P, \gamma)$ is a polynomial is easily established. For example, assuming that $P$ has $n$ elements, we have

$$
\Omega^{\prime}(P, \gamma)(m)=\sum_{k=1}^{n} c_{k}\left(\begin{array}{c}
m \\
k
\end{array}\right)
$$

where $c_{k}=c_{k}(P, \gamma)$ denotes the number of $f \in \mathcal{E}(P, \gamma)$ so that $\{|f(x)|: x \in X\}=$ $[k]$. In particular, $c_{n}$ counts the number of enriched $(P, \gamma)$-partitions in which each of $\pm 1, \ldots, \pm n$ occurs exactly once. Such partitions can be obtained by first choosing 
a linear extension of $(P, \gamma)$, and then choosing $n$ signs arbitrarily. Hence, a further consequence of (4.1) is the fact that

$$
\Omega^{\prime}(P, \gamma)(t)=\frac{2^{n}}{n !}|\mathcal{L}(P)| \cdot t^{n}+O\left(t^{n-1}\right) .
$$

The following result is the enriched analogue of (1.6).

Theorem 4.1. We have

$$
\sum_{m \geq 0} \Omega^{\prime}(P, \gamma)(m) t^{m}=\frac{1}{2} \frac{(1+t)^{n+1}}{(1-t)^{n+1}} \cdot W(P, \gamma)\left(\frac{4 t}{(1+t)^{2}}\right),
$$

where $W(P, \gamma)(t):=\sum_{w \in \mathcal{L}(P)} t^{1+|\Lambda(w, \gamma)|}$.

Proof. By linearity, it is enough to prove this for an arbitrarily labeled chain $([n], \gamma)$. For this we apply (1.6), obtaining

$$
\sum_{m \geq 0} L_{D}\left(1^{m}\right) t^{m}=\sum_{m \geq 0} \Omega([n], \gamma)(m) t^{m}=t^{1+|D|} /(1-t)^{n+1},
$$

where $D=D(w, \gamma)$ and $w=(1,2, \ldots, n)$ is the unique linear extension of the chain $[n]$. Thus by Proposition 3.5 , we have

$$
\sum_{m \geq 0} \Omega^{\prime}([n], \gamma)(m) t^{m}=\frac{2^{1+|\Lambda|} t}{(1-t)^{n+1}} \sum_{D \subset[n-1]: \Lambda \subset D \Delta(D+1)} t^{|D|},
$$

where $\Lambda=\Lambda(w, \gamma)$. We can analyze the sets $D$ appearing in the above sum as follows. For each $j \in \Lambda$, exactly one of $j$ or $j-1$ must belong to $D$; the remaining $n-2|\Lambda|-1$ elements of $[n-1]$ can be independently and arbitrarily included in $D$. Thus we have

$$
\begin{aligned}
\sum_{m \geq 0} \Omega^{\prime}([n], \gamma)(m) t^{m} & =\frac{2^{k+1} t}{(1-t)^{n+1}} \cdot(2 t)^{k}(1+t)^{n-2 k-1} \\
& =\frac{1}{2} \frac{(1+t)^{n+1}}{(1-t)^{n+1}} \cdot\left[\frac{4 t}{(1+t)^{2}}\right]^{k+1}
\end{aligned}
$$

where $k=|\Lambda([n], \gamma)|$.

In the theory of ordinary $P$-partitions, there is a Reciprocity Theorem (see [St1, $\S 10]$; cf. also Theorem 4.5.7 and Corollary 4.5.15 of [St2]) relating $(P, \gamma)$-partitions and $\left(P, \gamma^{*}\right)$-partitions. In terms of order polynomials, this reciprocity implies

$$
\Omega(P, \gamma)(t)=(-1)^{n} \Omega\left(P, \gamma^{*}\right)(-t) .
$$

In the enriched case, order polynomials enjoy self-reciprocity.

Proposition 4.2. We have $\Omega^{\prime}(P, \gamma)(t)=(-1)^{n} \Omega^{\prime}(P, \gamma)(-t)$; i.e., $\Omega^{\prime}(P, \gamma)(t)$ is either an even or an odd function of $t$.

Proof. If $F(t)=\sum_{m \geq 0} f(m) t^{m}$, where $f$ is a polynomial function of $m$, then one knows that $F(0)-F(1 / t)=\sum_{m \geq 0} f(-m) t^{m}$ as rational functions of $t$ (e.g., see Proposition 4.2.3 of [St2]). If we take $f=\Omega^{\prime}(P, \gamma)$, then $F(0)=f(0)=0$. Hence 
by Theorem 4.1,

$$
\begin{aligned}
\sum_{m \geq 0} \Omega^{\prime}(P, \gamma)(-m) t^{m} & =-\frac{1}{2} \frac{(1+1 / t)^{n+1}}{(1-1 / t)^{n+1}} \cdot W(P, \gamma)\left(\frac{4 / t}{(1+1 / t)^{2}}\right) \\
& =\frac{1}{2}(-1)^{n} \frac{(1+t)^{n+1}}{(1-t)^{n+1}} \cdot W(P, \gamma)\left(\frac{4 t}{(1+t)^{2}}\right) \\
& =(-1)^{n} \sum_{m \geq 0} \Omega^{\prime}(P, \gamma)(m) t^{m}
\end{aligned}
$$

and the result is evident.

4.2 A conjecture. The Neggers-Stanley Poset Conjecture [B] predicts that for every labeled poset $(P, \gamma)$, all zeroes of the polynomial $\sum_{w \in \mathcal{L}(P)} t^{1+|D(w, \gamma)|}$ are real. In view of (1.6), another formulation of this conjecture is that all zeroes of the rational function $\sum_{m>0} \Omega(P, \gamma)(m) t^{m}$ should be real. The following is an enriched analogue of the Neggers-Stanley conjecture.

Conjecture 4.3. For every labeled poset $(P, \gamma)$, all zeroes of $W(P, \gamma)(t)$ are real.

This conjecture would imply that the number of $w \in \mathcal{L}(P)$ such that $|\Lambda(w, \gamma)|=$ $k$ is log-concave with respect to $k$. We have verified the conjecture for all labeled posets on $\leq 7$ vertices, all naturally labeled posets on 8 vertices, and a few thousand randomly generated posets on $<20$ vertices.

With Theorem 4.1, the following result shows that Conjecture 4.3 is equivalent to the conjecture that all zeroes of the rational function $\sum_{m \geq 0} \Omega^{\prime}(P, \gamma)(m) t^{m}$ are real.

Proposition 4.4. If $f(t)$ is a polynomial with nonnegative (real) coefficients, then all zeroes of $f$ are real if and only if all zeroes of $g(t)=f\left(4 t /(1+t)^{2}\right)$ are real.

Proof. First, suppose that the zeroes of $g$ are real, and let $z$ be a zero of $f$. We can represent $z$ in the form $4 t /(1+t)^{2}$ for some complex $t$ by either solving the appropriate quadratic (if $z \neq 0$ ), or by taking $t=0$ (if $z=0$ ). Any such $t$ must therefore be a zero of $g$, and hence real, so $z$ must be real.

Conversely, suppose that the zeroes of $f$ are real, and let $t$ be a zero of $g$. Either we have $t=-1$, or else $4 t /(1+t)^{2}$ is a zero of $f$ and therefore real; we can dismiss the former case. Since the coefficients of $f$ are nonnegative, the zeroes of $f$ are nonpositive. Thus we can write $4 t /(1+t)^{2}=-z$ for some real $z>0$ (dismissing also the case $t=0)$. It follows that $0=4 t+z(1+t)^{2}=z t^{2}+(4+2 z) t+z$, a quadratic in $t$ with discriminant $(4+2 z)^{2}-4 z^{2}=16+16 z>0$. Hence $t$ must be real.

Consider the (non-linear) operator $f \mapsto W(f)$ on polynomials defined by

$$
\sum_{m \geq 0} f(m) t^{m}=W(f)(t) /(1-t)^{1+\operatorname{deg}(f)} .
$$

The following result is due to Wagner [W].

Theorem 4.5. If all zeroes of $W(f)$ and $W(g)$ are real and non-positive, then the same is true of $W(f g)$.

Corollary 4.6. If all zeroes of $W(P, \gamma)$ and $W(Q, \delta)$ are real, then the same is true of $W((P, \gamma)+(Q, \delta))$. In particular, Conjecture 4.3 is true for disjoint unions of arbitrarily labeled chains. 
A combinatorial consequence of this result is that the distribution of numbers of peaks over the set of shuffles of an arbitrary collection of (disjoint) permutations is log-concave.

Remark 4.7. If $f(t)$ is the order polynomial (ordinary or enriched) of a labeled poset, then by a result of Brenti (Theorem 4.4.1 in [B]), it follows that if all zeroes of $f$ are real, then the same is true of $W(f)$. This can provide a convenient means for proving instances of Conjecture 4.3 or its ordinary counterpart. For example, if $P=D_{\lambda}$ is a canonically labeled Young diagram, then $\Omega(P)(m)=s_{\lambda}\left(1^{m}\right)$, a polynomial whose zeroes are well-known to be all real (in fact, integral). On the other hand, if we consider enriched order polynomials of shifted diagrams $P=D_{\lambda}^{\prime}$, we no longer find that all zeroes of $\Omega^{\prime}(P)(m)$ are real (much less, integral). For example, if $\lambda=(3)$, then $P$ is a naturally labeled chain, and $\Omega^{\prime}(P)(m)=Q_{3}\left(1^{m}\right)=$ $2 m\left(2 m^{2}+1\right) / 3$. In fact $Q_{n}\left(1^{m}\right)$, the enriched order polynomial of a naturally labeled $n$-element chain, does not appear to factor significantly over $\mathbf{Q}$.

In view of Proposition 4.2, it is reasonable to ask if all zeroes of $\Omega^{\prime}\left(D_{\lambda}^{\prime}\right)(m)=$ $Q_{\lambda}\left(1^{m}\right)$ are real or purely imaginary. However this too is false, the smallest counterexample being $\lambda=(4,3)$, for which we have

$$
\Omega^{\prime}\left(D_{(4,3)}^{\prime}\right)(m)=Q_{43}\left(1^{m}\right)=\frac{4}{63} m\left(m^{2}-1\right)\left(2 m^{4}-5 m^{2}+9\right) .
$$

Remark 4.8. The "peak numbers" $P(n, k)=\left|\left\{w \in S_{n}:|\Lambda(w)|=k\right\}\right|$ (i.e., the number of permutations of $[n]$ having $k$ peaks, relative to the natural labeling) have several interesting combinatorial properties. By analyzing the placement of ' $n$ ', it is easy to establish the three-term recurrence

$$
P(n, k)=(2 k+2) P(n-1, k)+(n-2 k) P(n-1, k-1) .
$$

Note that $P(n, k)=0$ for $k \geq n / 2$, and that $P(2 n+1, n)$ is the number of "updown" permutations (also known as the tangent numbers). It is also evident that $P(n, 0)=2^{n-1}$ and $P(2 n+1, n)=P(2 n, n-1)$.

If we define $W_{n}(t)=\sum_{k} P(n, k) t^{k+1}$, then an equivalent formulation of (4.2) is

$$
W_{n}(t)=2 t(1-t) \frac{d}{d t} W_{n-1}(t)+n t W_{n-1}(t)
$$

for $n \geq 2$, with initial condition $W_{1}(t)=t$. Note that $W_{n}=W(P, \gamma)$ for an $n$ element antichain $P$. Since $\Omega^{\prime}(P, \gamma)(m)=(2 m)^{n}$ in this case, Theorem 4.1 implies

$$
\frac{1}{2} \frac{(1+t)^{n+1}}{(1-t)^{n+1}} \cdot W_{n}\left(\frac{4 t}{(1+t)^{2}}\right)=\sum_{m \geq 0}(2 m)^{n} t^{m}=\frac{2^{n} A_{n}(t)}{(1-t)^{n+1}}
$$

the second equality being a well-known property of the classical Eulerian polynomial $A_{n}(t)=\sum_{w \in S_{n}} t^{1+|D(w)|}$ (e.g., [C, p. 254]). Thus we have the unexpected relationship

$$
(1+t)^{n+1} W_{n}\left(4 t /(1+t)^{2}\right)=2^{n+1} A_{n}(t) .
$$

From Proposition 4.4 and the fact that all zeroes of $A_{n}(t)$ are real (e.g., Exercise 7.3 of [C]), it follows that all zeroes of $W_{n}(t)$ are real. (Of course, this is also a special case of Corollary 4.6.) In particular, the peak numbers $P(n, k)$ are log-concave with respect to $k$. 
As a final remark, we point out that the formal power series $\Delta(P, \gamma)\left(q, q^{2}, q^{3}, \ldots\right)$ appears to have no expansions with special combinatorial significance, in contrast to the case of ordinary $(P, \gamma)$-partitions (see (1.5)).

\section{Variations on a theme of Gessel}

In [G], Gessel notes some interesting applications of the theory of ordinary $P$ partitions that yield, for example, an easy combinatorial interpretation of some special Littlewood-Richardson coefficients and some combinatorial results essentially due to Foulkes and Foata-Schützenberger on the number of permutations $w$ with $D(w)=D$ and $D\left(w^{-1}\right)=E$ for fixed values of $D$ and $E$. In this section, we show that there are similar applications of the theory of enriched $P$-partitions. In particular, we give a (new) combinatorial interpretation of some special shifted Littlewood-Richardson coefficients (Section 5.1), and a variation on the Foulkes and Foata-Schützenberger results (Section 5.2).

5.1 Symmetric functions and descent sets. Let $n$ be a fixed positive integer. For $D \subset[n-1]$, define $N_{D}=([n], \unlhd)$ to be the partial order obtained from the transitive closure of the relations

$$
i \triangleleft i+1 \text {, if } i \notin D ; \quad i+1 \triangleleft i, \text { if } i \in D .
$$

As a labeling of $N_{D}$, we will use the identity map $\iota$, with the label set $[n]$ being totally ordered in the usual way. For permutations $w$ of $[n]$, we will write $D(w)$ as an abbreviation for $D(w, \iota)$. Figure 5 provides an illustration of the labeled poset $\left(N_{D}, \iota\right)$ in the case $n=9, D=\{3,7,8\}$. It is clear that $\left(N_{D}, \iota\right)$ is isomorphic to a canonically labeled (unshifted) skew diagram. ${ }^{4}$ Hence $\Gamma\left(N_{D}, \iota\right)$ is a skew Schur function; although it is an abuse of notation, we will write $s_{D}=\Gamma\left(N_{D}, \iota\right)$ and $S_{D}=\theta s_{D}=\Delta\left(N_{D}, \iota\right)$.

From the definition of $N_{D}$, it is clear that a permutation $w \in S_{n}$ is a linear extension of $N_{D}$ precisely if $i+1$ precedes $i$ in $\left(w_{1}, \ldots, w_{n}\right)$ if and only if $i \in D$. In

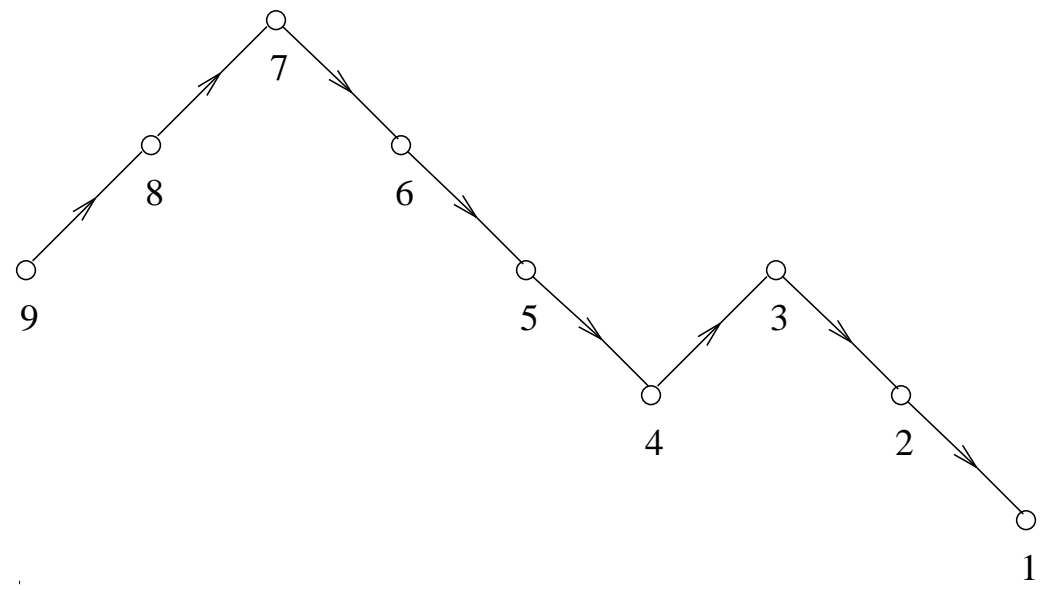

Figure 5. An oriented border strip.

\footnotetext{
${ }^{4}$ The diagram that arise in this fashion are known in the literature as border strips, or rim hooks.
} 
other words,

$$
\mathcal{L}\left(N_{D}\right)=\left\{w \in S_{n}: D\left(w^{-1}\right)=D\right\} .
$$

The following is Theorem 3 of $[\mathrm{G}]$; we include below a slightly different proof.

Lemma 5.1. For any $f \in \boldsymbol{\Lambda}^{n},\left\langle f, s_{D}\right\rangle$ is the coefficient of $L_{D}$ in $f$ as a member of $\boldsymbol{\Sigma}^{n}$.

Proof. It suffices to prove the assertion for $f=h_{\lambda}$, where $\lambda=\left(\lambda_{1}, \ldots, \lambda_{l}\right)$ is an arbitrary partition of $n$. For this, let $E_{\lambda}=\left\{a_{1}, \ldots, a_{l-1}\right\}$, where $a_{j}=\lambda_{1}+\cdots+\lambda_{j}$. Since the monomial symmetric functions $m_{\lambda}$ are dual to the $h_{\lambda}$ 's, it follows that $\left\langle h_{\lambda}, s_{D}\right\rangle$ is the coefficient of $m_{\lambda}$ in $s_{D}$, or equivalently, the coefficient of $M_{E}$ in $s_{D}$ (as a member of $\boldsymbol{\Sigma}^{n}$ ). However by (1.7) and (5.1), we have

$$
s_{D}=\sum_{w: D\left(w^{-1}\right)=D} L_{D(w)}=\sum_{w: D\left(w^{-1}\right)=D, D(w) \subset E} M_{E},
$$

so $\left\langle h_{\lambda}, s_{D}\right\rangle=\left|\left\{w \in S_{n}: D\left(w^{-1}\right)=D, D(w) \subset E_{\lambda}\right\}\right|$.

On the other hand, we have $h_{\lambda}=\Gamma\left(C_{\lambda}, \iota\right)$, where $C_{\lambda}$ denotes the order-theoretic union of the chains $a_{j}+1<\cdots<a_{j+1}\left(0 \leq j \leq l-1, a_{0}=0, a_{l}=n\right)$. Note also that

$$
\mathcal{L}\left(C_{\lambda}\right)=\left\{w \in S_{n}: D\left(w^{-1}\right) \subset E_{\lambda}\right\} .
$$

Hence by (1.7), the coefficient of $L_{D}$ in $h_{\lambda}$ equals

$$
\left|\left\{w \in S_{n}: D\left(w^{-1}\right) \subset E_{\lambda}, D(w)=D\right\}\right|,
$$

which agrees with the previous computation of $\left\langle h_{\lambda}, s_{D}\right\rangle$.

As an application, consider $f=\Gamma\left(D_{\lambda / \mu}\right)=s_{\lambda / \mu}$. The above result implies that $\left\langle s_{\lambda / \mu}, s_{D}\right\rangle$ is the number of standard tableaux $T \in \mathcal{F}^{\lambda / \mu}$ such that $D(T)=D$. In the case $\mu=\emptyset$, this can be viewed as a special case of the Littlewood-Richardson rule. Conversely, it is routine to deduce Lemma 5.1 from the Littlewood-Richardson rule.

More generally, if $f$ is a symmetric function such that $f=\Gamma(P, \gamma)$ for some labeled poset $(P, \gamma)$, Lemma 5.1 provides a combinatorial interpretation of the scalar product $\left\langle f, s_{D}\right\rangle$. However, this is probably a vacuous generalization, since Stanley has conjectured that the only $\Gamma$-symmetric posets (i.e., labeled posets $(P, \gamma)$ such that $\Gamma(P, \gamma)$ is symmetric) are canonically labeled (unshifted) skew diagrams [St1, $\S 21]$. (See also Theorem 4.5 of [St3] for some recent evidence in support of this conjecture.)

Turning to the enriched analogues of these results, let us first recall that by Corollary 3.3 , every $\Gamma$-symmetric poset is also $\Delta$-symmetric. On the other hand, we certainly know that all canonically labeled shifted skew diagrams are $\Delta$-symmetric, so the class of $\Delta$-symmetric posets is strictly larger than the class of $\Gamma$-symmetric posets. Let us also recall from Theorem 3.8 that if $f$ is a symmetric function such that $f=\Delta(P, \gamma)$ for some labeled poset $(P, \gamma)$, then $f \in \boldsymbol{\Omega}$. In that case, the following result provides a combinatorial interpretation for $\left[f, S_{D}\right]$, where $[\cdot, \cdot]$ denotes the usual scalar product one has in $\boldsymbol{\Omega}_{\mathrm{Q}}$ (see Section A.4).

Theorem 5.2. Let $D \subset[n-1]$. If $(P, \gamma)$ is a positively labeled $\Delta$-symmetric partial order of size $n$, then $\left[\Delta(P, \gamma), S_{D}\right]$ is the coefficient of $L_{D}$ in $\Delta(P, \gamma)$. Moreover,

$$
\left[\Delta(P, \gamma), S_{D}\right]=\left|\left\{(\varepsilon, w) \in\{ \pm 1\}^{n} \times \mathcal{L}(P): D(w, \varepsilon \gamma)=D\right\}\right| .
$$



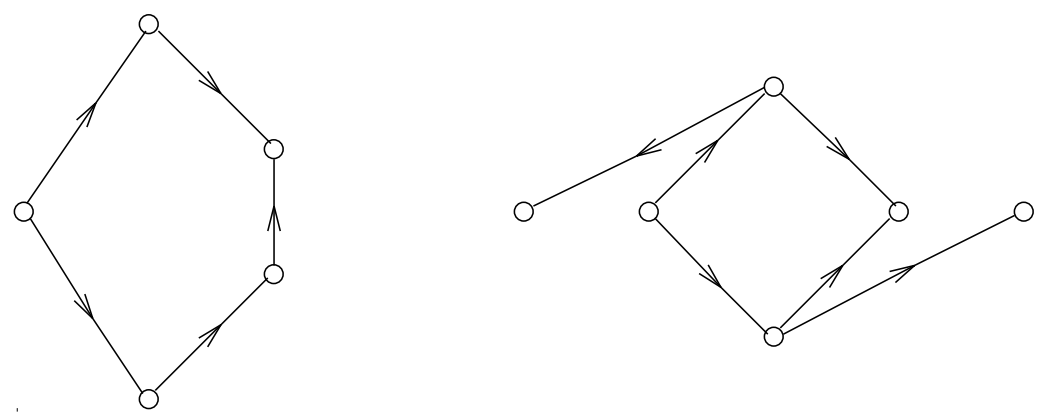

Figure 6 . Two $\Delta$-symmetric posets.

Proof. By Lemma 5.1 and (A.9), it follows that $\left[\Delta(P, \gamma), S_{D}\right]$ is the coefficient of $L_{D}$ in $\Delta(P, \gamma)$. By Theorem 3.6 and (1.7), it follows that this coefficient has the claimed interpretation.

As an application, let $(P, \gamma)$ be a canonically labeled shifted skew diagram $D_{\lambda / \mu}^{\prime}$, so that $\Delta(P, \gamma)=Q_{\lambda / \mu}$. We can identify $\{ \pm 1\}^{n} \times \mathcal{L}(P)$ with the set $\mathcal{G}_{ \pm}^{\lambda / \mu}$ consisting of all shifted $\mathbf{P}^{\prime}$-tableaux of shape $\lambda / \mu$ in which exactly one of $j$ or $-j$ occurs for $1 \leq j \leq n$. If $T$ is the tableau corresponding to some pair $(\varepsilon, w)$ under this identification, then the descent set $D(T)$ should be defined so as to agree with $D(w, \varepsilon \gamma)$; this yields

$$
D(T)=\left\{i \in D(|T|): \varepsilon_{i}=+1\right\} \cup\left\{i \notin D(|T|): \varepsilon_{i+1}=-1\right\},
$$

where $\varepsilon_{i}=-1$ iff $-i$ is an entry of $T$, and $|T| \in \mathcal{G}^{\lambda / \mu}$ denotes the standard tableau corresponding to $T$. Applying Theorem 5.2, we obtain

$$
\left[Q_{\lambda / \mu}, S_{D}\right]=\left|\left\{T \in \mathcal{G}_{ \pm}^{\lambda / \mu}: D(T)=D\right\}\right|
$$

We note that $S_{D}$ is also a skew $Q$-function, so at least in the case $\mu=\emptyset$, this can be viewed as an interpretation of certain shifted Littlewood-Richardson coefficients. However, to deduce this interpretation from the shifted analogue of the LittlewoodRichardson rule [Ste1, §8] does not appear to be straightforward.

In view of Stanley's conjecture on $\Gamma$-symmetric posets, it is natural to ask if this is the only application of Theorem 5.2; i.e., are canonically labeled shifted skew diagrams the only $\Delta$-symmetric posets? Interestingly, the answer turns out to be resoundingly negative. As we will demonstrate in a sequel to this paper, the class of $\Delta$-symmetric posets is extremely complicated, and in any case includes many posets that are not shifted skew diagrams, such as the two labeled posets in Figure 6. An even more obvious discrepancy is the fact that the class of $\Delta$-symmetric posets is closed under disjoint union, whereas the class of shifted skew diagrams is not.

5.2 Strange symmetries. For $D \subset[n-1]$, let $D^{c}=[n-1]-D$ and $\bar{D}=\{n-i$ : $i \in D\}$.

Proposition 5.3. If $(P, \gamma)$ is a positively labeled $\Delta$-symmetric partial order of size $n$, then the quantities

$$
c_{D}(P, \gamma)=\left|\left\{(\varepsilon, w) \in\{ \pm 1\}^{n} \times \mathcal{L}(P): D(w, \varepsilon \gamma)=D\right\}\right|
$$

are invariant under the transformations $D \mapsto D^{c}$ and $D \mapsto \bar{D}$. 
Proof. If $(Q, \delta)$ is $\Delta$-symmetric, then $\Delta(Q, \delta)=\Delta\left(Q^{*}, \delta\right)$, by (2.3). In particular,

$$
S_{D}=\Delta\left(N_{D}, \iota\right)=\Delta\left(N_{D}^{*}, \iota\right)=S_{D^{c}} .
$$

Similarly, $\left(N_{D}, \iota\right)$ is also $\Gamma$-symmetric, so by $(1.2)$ we have

$$
s_{D}=\Gamma\left(N_{D}, \iota\right)=\Gamma\left(N_{D}^{*}, \iota^{*}\right)=s_{\bar{D}} .
$$

Hence $S_{D}=S_{D^{c}}=S_{\bar{D}}$, and the result follows from Theorem 5.2.

For example, if we take $(P, \gamma)=\left(N_{E}, \iota\right)$ for some $E \subset[n-1]$, we obtain that the quantity

$$
\left[S_{D}, S_{E}\right]=\left|\left\{(\varepsilon, w) \in\{ \pm 1\}^{n} \times S_{n}: D\left(w^{-1}\right)=E, D(w, \varepsilon \iota)=D\right\}\right|
$$

is invariant under the transformations $D \mapsto D^{c}, D \mapsto \bar{D}$, and $(D, E) \mapsto(E, D)$; these in turn generate a group of 32 transformations. We regard the identity implied by (5.2) as an analogue of the fact (Theorem 5 of $[\mathrm{G}]$ ) that

$$
\left\langle s_{D}, s_{E}\right\rangle=\left|\left\{w \in S_{n}: D\left(w^{-1}\right)=E, D(w)=D\right\}\right| .
$$

Continuing the analogy, the fact that $s_{D}=s_{\bar{D}}$ shows that this quantity is invariant under the transformation $D \mapsto \bar{D}$, a result Gessel attributes to Foata and Schützenberger (see Corollary 6 of $[\mathrm{G}]$ ).

5.3 Symmetric functions and peak sets. As in Section $5.1, n$ is a fixed positive integer. Returning to Lemma 5.1, observe that one could define $s_{D}$ as the unique symmetric function with the property that for all $f \in \Lambda^{n},\left\langle f, s_{D}\right\rangle$ is the coefficient of $L_{D}$ in $f$. Thus by analogy it is natural to define a symmetric function $Z_{\Lambda} \in \Omega_{\mathbf{Q}}^{n}$ for each peak set $\Lambda$ so that for all $f \in \boldsymbol{\Omega}_{\mathbf{Q}}^{n}$,

$$
\left[f, Z_{\Lambda}\right]=\text { the coefficient of } K_{\Lambda} \text { in } f \text {. }
$$

Proposition 5.4. We have

(a) $Z_{\Lambda}=\sum_{\lambda} b_{\Lambda, \lambda} P_{\lambda}$, where $b_{\Lambda, \lambda}=\left|\left\{T \in \mathcal{G}^{\lambda}: \Lambda(T)=\Lambda\right\}\right|$.

(b) $Z_{\Lambda}=\sum_{w \in S_{n}: \Lambda\left(w^{-1}\right)=\Lambda} L_{D(w)}$.

(c) $Z_{\Lambda}=\sum_{D \subset[n-1]: \Lambda(D)=\Lambda} s_{D}$, where $\Lambda(D)=\{i \geq 2: i \in D, i-1 \notin D\}$.

Proof. By (2.5), we have that $Q_{\lambda}=\sum_{T \in \mathcal{G}^{\lambda}} K_{\Lambda(T)}$, so $\left[Q_{\lambda}, Z_{\Lambda}\right]=b_{\Lambda, \lambda}$. Hence by (A.6), this quantity must be the coefficient of $P_{\lambda}$ in $Z_{\Lambda}$, which proves (a).

For (b), choose $D \subset[n-1]$. By Lemma $5.1,\left\langle Z_{\Lambda}, s_{D}\right\rangle$ is the coefficient of $L_{D}$ in $Z_{\Lambda}$. On the other hand, by (A.9), we have $\left\langle Z_{\Lambda}, s_{D}\right\rangle=\left[Z_{\Lambda}, S_{D}\right]$, so this quantity is also the coefficient of $K_{\Lambda}$ in $S_{D}=\Delta\left(N_{D}, \iota\right)$. However by (5.1) and (2.4), we have

$$
S_{D}=\sum_{w: D\left(w^{-1}\right)=D} K_{\Lambda(w)}
$$

so this coefficient is $\left|\left\{w \in S_{n}: D\left(w^{-1}\right)=D, \Lambda(w)=\Lambda\right\}\right|$.

One can prove (c) by first noting that $\Lambda(w)$ depends only on $D(w)$, and then collecting together the terms of (b) corresponding to a given value of $D\left(w^{-1}\right)$.

We remark that the above expansions show that $Z_{\Lambda} \in \overline{\mathbf{\Omega}}$.

Keeping in mind the analogy between $s_{D}$ and $Z_{\Lambda}$, it is natural to ask if $Z_{\Lambda}$ is a skew $P$-function, or more generally, if there exists a labeled poset $(P, \gamma)$ such that $Z_{\Lambda}=\Delta(P, \gamma)$ (or some scalar multiple thereof). However, this turns out to 
be false. For example, if $n=7$ and $\Lambda=\{2,4,6\}$, then $Z_{\Lambda}=P_{43}+P_{421}$ (by Proposition 5.4(a)), and it can be shown that there is no poset $(P, \gamma)$ such that $\Delta(P, \gamma)=c\left(P_{43}+P_{421}\right)$, for any scalar $c$.

There do exist simple formulas for several special instances of $Z_{\Lambda}$.

Proposition 5.5. We have

(a) $Z_{\varnothing}=P_{(n)}$.

(b) $Z_{\{2\}}=Z_{\{n-1\}}=P_{(n-1,1)}$, if $n \geq 3$.

(c) $Z_{\{2, n-1\}}=P_{(n-2,2)}$, if $n \geq 5$.

(d) $Z_{\{2,4, \ldots, 2 m\}}=s_{(m+1, \ldots, 1) /(m-1, \ldots, 1)}$, if $n=2 m+1$.

(e) $Z_{\Lambda}=Z_{\bar{\Lambda}}$.

Proof. Parts (a), (b), and (c) follow from Proposition 5.4(a) and the fact that there is a unique standard shifted tableau with peak set $\Lambda$, for each of $\Lambda=\varnothing$, $\{2\},\{n-1\}$, and $\{2, n-1\}$. Part (d) follows from Proposition 5.4(c) and the fact $D=\{2,4, \ldots, 2 m\}$ is the unique descent set in $[2 m]$ whose peak set is $\{2,4, \ldots, 2 m\}$. For $(\mathrm{e})$, note that for any labeled poset $(P, \gamma)$, we have $\left(w_{1}, \ldots, w_{n}\right) \in \mathcal{L}(P)$ if and only if $\left(w_{n}, \ldots, w_{1}\right) \in \mathcal{L}\left(P^{*}\right)$, so the coefficient of $K_{\Lambda}$ in $\Delta(P, \gamma)$ equals the coefficient of $K_{\bar{\Lambda}}$ in $\Delta\left(P^{*}, \gamma\right)$. Therefore if $(P, \gamma)$ is $\Delta$-symmetric, then $\left[\Delta(P, \gamma), Z_{\Lambda}\right]=$ $\left[\Delta(P, \gamma), Z_{\bar{\Lambda}}\right]$, by $(2.3)$. In particular $\left[Q_{\lambda}, Z_{\Lambda}\right]=\left[Q_{\lambda}, Z_{\bar{\Lambda}}\right]$ for all strict partitions $\lambda$.

Let $a_{n}(\Lambda)$ denote the number of permutations in $S_{n}$ with peak set $\Lambda$. We have $a_{n}(\Lambda)=\left[q_{1}^{n}, Z_{\Lambda}\right]$, since $q_{1}^{n}$ is the enriched weight enumerator of an $n$-element antichain. On the other hand, by (A.7) one knows that $\left[q_{1}^{n}, P_{\lambda}\right]=2^{n-\ell(\lambda)} \cdot\left|\mathcal{G}^{\lambda}\right|$, so

$$
a_{n}(\Lambda)=\sum_{\lambda} 2^{n-\ell(\lambda)} b_{\Lambda, \lambda}\left|\mathcal{G}^{\lambda}\right|,
$$

where $b_{\Lambda, \lambda}$ denotes the transition matrix defined in Proposition 5.4(a). Thus a modest application of Proposition 5.5 yields the formulas $a_{n}(\varnothing)=2^{n-1}, a_{n}(\{2\})=$ $2^{n-2}(n-2)$, and $a_{n}(\{2, n-1\})=2^{n-3}(n-1)(n-4)$. At least the first of these is well-known; it was noted previously in Remark 4.8 .

\section{APPENDIX: On $Q$-FUnCTIONS}

In this Appendix, we survey some of the fundamental properties of Schur's $Q$ functions, as found in $[\mathrm{HH}],[\mathrm{J}],[\mathrm{JP}]$, Chapter III of $[\mathrm{M}],[\mathrm{P}],[\mathrm{Sa}]$, and $[\mathrm{Ste} 1$, Ste2, Ste3].

We assume familiarity with the notation for symmetric functions in Chapter I of [M]. In particular, $e_{n}, h_{n}$, and $p_{n}$ denote (respectively) the $n$th elementary, complete homogeneous, and power-sum symmetric functions, and $m_{\lambda}$ and $s_{\lambda}$ denote the monomial and Schur functions indexed by the partition $\lambda$. We use the notation $\boldsymbol{\Lambda}=\bigoplus_{n \geq 0} \boldsymbol{\Lambda}^{n}$ for the graded ring of symmetric functions in the variables $z_{1}, z_{2}, \ldots$, with integer coefficients.

A.1 Definitions. For integers $n \geq 0$, define symmetric functions $q_{n} \in \boldsymbol{\Lambda}^{n}$ by setting

$$
Q(t)=\sum_{n \geq 0} q_{n} t^{n}=\prod_{i \geq 1} \frac{1+z_{i} t}{1-z_{i} t}
$$


For pairs of integers $m, n \geq 0$, define $Q_{(m, n)} \in \boldsymbol{\Lambda}^{m+n}$ by setting

$$
\left(Q\left(t_{1}\right) Q\left(t_{2}\right)-1\right) \frac{t_{1}-t_{2}}{t_{1}+t_{2}}=\sum_{m, n \geq 0} Q_{(m, n)} t_{1}^{m} t_{2}^{n} .
$$

Note that $Q_{(m, n)}=-Q_{(n, m)}$ and $Q_{(n, 0)}=q_{n}$ (if $\left.n>0\right)$.

Now let $\lambda=\left(\lambda_{1}>\cdots>\lambda_{l}>0\right)$ be a strict partition. If $l$ is odd, set $\lambda_{l+1}=0$, and replace $l$ by $l+1$. Thus in all cases, $l$ is even. The $Q$-function indexed by $\lambda$, as defined by Schur in [S], is given by

$$
Q_{\lambda}=\operatorname{Pf}\left[Q_{\left(\lambda_{i}, \lambda_{j}\right)}\right]_{1 \leq i, j \leq l} .
$$

More generally, the skew $Q$-function $Q_{\lambda / \mu}$ can also be defined as the Pfaffian of a matrix whose nonzero entries are $Q_{(m, n)}$ 's $[\mathrm{JP}]$.

For any shifted skew shape $\lambda / \mu$, a shifted $\mathbf{P}^{\prime}$-tableau $T$ of shape $\lambda / \mu$ is (as in Section 2.4) an enriched $D_{\lambda / \mu}^{\prime}$-partition, i.e., an order-preserving map $T: D_{\lambda / \mu}^{\prime} \rightarrow$ $\mathbf{P}^{\prime}$ such that the positive (resp., negative) entries in each column (resp., row) are distinct. For example, the following is a shifted $\mathbf{P}^{\prime}$-tableau of shape $7421 / 1$, using the traditional English-style presentation of tableaux:

$$
\begin{array}{llllll}
-1 & +1 & -2 & -3 & +5 & +5 \\
+2 & +2 & -3 & +3 & & \\
& +3 & +3 & & & \\
& & & & & \\
& & & & &
\end{array}
$$

The skew $Q$-functions may also be defined as the generating function for shifted $\mathbf{P}^{\prime}$-tableaux; i.e.,

$$
Q_{\lambda / \mu}=\sum_{T: D_{\lambda / \mu}^{\prime} \rightarrow \mathbf{P}^{\prime}} z_{1}^{\alpha_{1}(T)} z_{2}^{\alpha_{2}(T)} \cdots,
$$

where $\alpha_{j}(T)=\left|T^{-1}(j)\right|+\left|T^{-1}(-j)\right|$. A direct proof of the equivalence of this definition with Schur's definition can be found in [Ste3, §6].

A third definition of the (skew) $Q$-functions may be obtained by setting $Q_{\lambda / \mu}(z)$ $=Q_{\lambda / \mu}(z ;-1)$, where $Q_{\lambda / \mu}(z ; t)$ denotes the Hall-Littlewood function indexed by $\lambda / \mu$. A proof of the equivalence of this definition with the tableau definition can be deduced from [M, III.(5.11)]; a direct proof of the equivalence with Schur's definition can be found in $[\mathrm{J}]$, for example.

A.2 The $Q$-function algebra. Let $\boldsymbol{\Omega}=\bigoplus_{n \geq 0} \boldsymbol{\Omega}^{n}$ denote the graded subring of $\boldsymbol{\Lambda}$ generated by $1, q_{1}, q_{2}, \ldots$ It is clear from Schur's definition that $Q_{\lambda} \in \boldsymbol{\Omega}$, and more generally from the Józefiak-Pragacz Pfaffian that $Q_{\lambda / \mu} \in \boldsymbol{\Omega}$. By logarithmically differentiating (A.1), we obtain

$$
\frac{d}{d t} Q(t)=Q(t) \sum_{r \geq 0} 2 p_{2 r+1} t^{2 r+1}
$$

or equivalently,

$$
n q_{n}=\sum_{r \geq 0} 2 p_{2 r+1} q_{n-2 r-1} .
$$

It follows that $2 p_{2 n+1} \in \mathbf{\Omega}$, and that if we pass to the rational field, the $\mathbf{Q}$-algebra $\boldsymbol{\Omega}_{\mathbf{Q}}=\mathbf{Q} \otimes_{\mathbf{Z}} \boldsymbol{\Omega}$ is freely generated by $p_{1}, p_{3}, p_{5}, \ldots ;$ i.e.,

$$
\mathbf{\Omega}_{\mathbf{Q}} \cong \mathbf{Q}\left[p_{1}, p_{3}, p_{5}, \ldots\right] \text {. }
$$


Expressing (A.2) as a symmetric function expansion, we have

$$
Q_{\lambda / \mu}=\sum_{\alpha} K_{\lambda / \mu, \alpha}^{\prime} m_{\alpha}
$$

where $\alpha$ ranges over partitions, and $K_{\lambda / \mu, \alpha}^{\prime}$ denotes the number of shifted $\mathbf{P}^{\prime}$ tableaux $T$ of shape $\lambda / \mu$ such that $\alpha_{j}(T)=\alpha_{j}$. It turns out that the transition matrix $\left[K_{\lambda, \alpha}^{\prime}\right]$ also appears in the expansion

$$
q_{\alpha_{1}} q_{\alpha_{2}} \cdots=\sum_{\lambda} 2^{-\ell(\lambda)} K_{\lambda, \alpha}^{\prime} Q_{\lambda}
$$

where the sum ranges over strict partitions $\lambda$ (Cor. 6.2 of [Ste1]). It is also not hard to show that the (rectangular) matrix $\left[2^{-\ell(\lambda)} K_{\lambda, \alpha}^{\prime}\right]$ is (a) upper unitriangular and (b) integral (Prop. 6.3 of [Ste1]), which allows us to conclude that the $Q_{\lambda}$ 's (a) are linearly independent over $\mathbf{Q}$ (by (A.3)), and (b) form a free $\mathbf{Z}$-basis of $\boldsymbol{\Omega}$ (by (A.4)).

We remark that there is a surjective ring homomorphism $\theta: \boldsymbol{\Lambda} \rightarrow \boldsymbol{\Omega}$ satisfying

$$
\theta\left(e_{n}\right)=\theta\left(h_{n}\right)=q_{n}, \quad \theta\left(p_{n}\right)=\left\{\begin{array}{cl}
2 p_{n} & \text { if } n \text { is odd } \\
0 & \text { if } n \text { is even }
\end{array}\right.
$$

A.3 The $P$-function algebra. The $\mathbf{Q}$-algebra $\boldsymbol{\Omega}_{\mathbf{Q}}$ has a second important graded subring; namely,

$$
\bar{\Omega}:=\Omega_{\mathbf{Q}} \cap \Lambda .
$$

The members of $\overline{\boldsymbol{\Omega}}$ are thus the symmetric functions in $\boldsymbol{\Omega}_{\mathrm{Q}}$ that have integer coefficients relative to the variables $z_{1}, z_{2}, \ldots$; this ring is strictly larger than $\boldsymbol{\Omega}$, since $p_{1} \notin \boldsymbol{\Omega}$.

Fix a shifted skew shape $\lambda / \mu$, and let $(i, i) \in D_{\lambda / \mu}^{\prime}$ be one of the $\ell(\lambda)-\ell(\mu)$ cells of $\lambda / \mu$ on the main diagonal. If $T$ is any shifted $\mathbf{P}^{\prime}$-tableau of shape $\lambda / \mu$, then so too is the tableau obtained by substituting $T(i, i)=-T(i, i)$. It follows that $K_{\lambda / \mu, \alpha}^{\prime}$ is divisible by $2^{\ell(\lambda)-\ell(\mu)}$, and hence as a consequence of (A.2) or (A.3), the symmetric functions

$$
P_{\lambda / \mu}:=2^{\ell(\mu)-\ell(\lambda)} Q_{\lambda / \mu}
$$

belong to $\overline{\boldsymbol{\Omega}}$. Furthermore, since we know that the matrix $\left[2^{-\ell(\lambda)} K_{\lambda, \alpha}^{\prime}\right]$ is upper unitriangular, it follows from (A.3) that the $P_{\lambda}$ 's are a free $\mathbf{Z}$-basis of $\overline{\boldsymbol{\Omega}}$.

We remark that it is known (Theorem 2.11 of $[\mathrm{P}]$ ) that the ring $\overline{\boldsymbol{\Omega}}$ can be presented in terms of a "cancellation law;" namely,

$$
\overline{\mathbf{\Omega}}=\left\{f \in \boldsymbol{\Lambda}: f\left(t,-t, z_{3}, z_{4}, \ldots\right)=f\left(z_{3}, z_{4}, \ldots\right)\right\} .
$$

This cancellation law is often the simplest method for testing when a symmetric function belongs to the integral span of the $P_{\lambda}$ 's or the rational span of the $Q_{\lambda}$ 's. Note however that since $\boldsymbol{\Omega}$ is strictly contained in $\overline{\boldsymbol{\Omega}}$, asserting that a symmetric function $f$ is " $Q$-integral" is stronger than, and therefore preferable to, asserting that $f$ is "P-integral." 
A.4 Orthogonality. There is a Cauchy-type series identity for the $Q$-functions; viz.,

$$
\prod_{i, j \geq 1} \frac{1+x_{i} y_{j}}{1-x_{i} y_{j}}=\sum_{\lambda} P_{\lambda}(x) Q_{\lambda}(y)
$$

where $\lambda$ ranges over all strict partitions. A purely combinatorial proof, using the tableau definition of the $Q$-functions, has been given by Sagan [Sa]; an algebraic proof can be obtained by specializing the corresponding Hall-Littlewood series [M, III.(4.4)].

Several other expansions of the above series are possible, including

$$
\prod_{i, j \geq 1} \frac{1+x_{i} y_{j}}{1-x_{i} y_{j}}=\sum_{\mu} \frac{2^{\ell(\mu)}}{z_{\mu}} p_{\mu}(x) p_{\mu}(y)=\sum_{\lambda} q_{\lambda}(x) m_{\lambda}(y)=\sum_{\lambda} s_{\lambda}(x) S_{\lambda}(y),
$$

where $\mu$ (resp., $\lambda$ ) ranges over partitions with odd parts (resp., all partitions) and $S_{\lambda}=\theta s_{\lambda}$. See Props. 5.2, 5.6, and 9.2 of [Ste1], for example.

If we define a positive definite inner product on $\boldsymbol{\Omega}_{\mathrm{Q}}$ by setting

$$
\left[p_{\mu}, p_{\nu}\right]:=z_{\mu} 2^{-\ell(\mu)} \delta_{\mu, \nu}
$$

for all partitions $\mu, \nu$ with only odd parts, then as purely formal consequences of the above expansions (ibid.), we have

$$
\begin{aligned}
{\left[P_{\lambda}, Q_{\mu}\right] } & =\delta_{\lambda, \mu}, \\
{\left[f, q_{\lambda}\right] } & =\text { the coefficient of } m_{\lambda} \text { in } f, \\
{\left[f, S_{\lambda}\right] } & =\text { the coefficient of } s_{\lambda} \text { in } f,
\end{aligned}
$$

for all $f \in \boldsymbol{\Omega}_{\mathbf{Q}}$. By linearity, a common generalization of (A.7) and (A.8) is the identity

$$
\langle f, g\rangle=[f, \theta(g)]
$$

for all $f \in \boldsymbol{\Omega}_{\mathbf{Q}}, g \in \boldsymbol{\Lambda}_{\mathbf{Q}}$, where $\langle\cdot, \cdot\rangle$ denotes the standard inner product on $\boldsymbol{\Lambda}_{\mathbf{Q}}$; i.e.,

$$
\left\langle s_{\lambda}, s_{\mu}\right\rangle=z_{\lambda}^{-1}\left\langle p_{\lambda}, p_{\mu}\right\rangle=\left\langle h_{\lambda}, m_{\mu}\right\rangle=\delta_{\lambda, \mu} .
$$

\section{REFERENCES}

[B] F. Brenti, Unimodal, log-concave and Polya frequency sequences in combinatorics, Mem. Amer. Math. Soc. (1989), no. 413. MR 90d:05014

[C] L. Comtet, Advanced Combinatorics, Reidel, Dordrecht, 1974. MR 57:124

[G] I. M. Gessel, Multipartite P-partitions and inner products of skew Schur functions, Contemporary Math. 34 (1984), 289-301. MR 86k:05007

$[\mathrm{HH}]$ P. N. Hoffman and J. F. Humphreys, Projective representations of the symmetric groups, Oxford Univ. Press, Oxford, 1992. MR 94f: 20027

[J] T. Józefiak, Characters of projective representations of symmetric groups, Exposition. Math. 7 (1989), 193-247. MR 90f:20018

[JP] T. Józefiak and P. Pragacz, A determinantal formula for skew Schur Q-functions, J. London Math. Soc. 43 (1991), 76-90. MR 92d:05175

[M] I. G. Macdonald, Symmetric Functions and Hall Polynomials, Oxford Univ. Press, Oxford, 1979. MR 84g:05003

[P] P. Pragacz, Algebro-geometric applications of Schur $S$ - and Q-polynomials, in Topics in Invariant Theory (M.-P. Malliavin, ed.), pp. 130-191, Lecture Notes in Math. Vol. 1478, Springer-Verlag, Berlin, 1991. MR 93h:05170 
[Sa] B. E. Sagan, Shifted tableaux, Schur Q-functions and a conjecture of R. Stanley, J. Combin. Theory Ser. A 45 (1987), 62-103. MR 88f:05011

[S] I. Schur, Über die Darstellung der symmetrischen und der alternierenden Gruppe durch gebrochene lineare Substitutionen, J. Reine Angew. Math. 139 (1911), 155-250.

[St1] R. P. Stanley, Ordered structures and partitions, Mem. Amer. Math. Soc. (1972), no. 119. MR 48:10836

[St2] R. P. Stanley, Enumerative Combinatorics, Vol. I," Wadsworth \& Brooks/Cole, Monterey, 1986. MR 87j:05003

[St3] R. P. Stanley, Flag-symmetric and locally rank-symmetric partially ordered sets, Electron. J. Combin. 3 (1996), Research Paper 6.

[Ste1] J. R. Stembridge, Shifted tableaux and the projective representations of symmetric groups, Adv. in Math. 74 (1989), 87-134. MR 90k:20026

[Ste2] J. R. Stembridge, On symmetric functions and the spin characters of $S_{n}$, in "Topics in Algebra," (S. Balcerzyk et al., eds.), Banach Center Publ. Vol. 26, part 2, pp. 433-453, Polish Scientific Publishers, Warsaw, 1990. MR 93e:20018

[Ste3] J. R. Stembridge, Nonintersecting paths, pfaffians and plane partitions, Adv. in Math. 83 (1990), 96-131. MR 91h:05014

[W] D. G. Wagner, Total positivity of Hadamard products, J. Math. Anal. Appl. 163 (1992), 459-483. MR 93f: 15020

Department of Mathematics, University of Michigan, Ann Arbor, Michigan 481091109 